\title{
Quasiprobability Distribution for Heat Fluctuations in the Quantum Regime
}

\author{
Amikam Levy $\oplus^{1,2,3,{ }^{*}}$ and Matteo Lostaglio $\oplus^{4,5, \uparrow}$ \\ ${ }^{1}$ Department of Chemistry, University of California, Berkeley, California 94720, USA \\ ${ }^{2}$ The Raymond and Beverly Sackler Center for Computational Molecular and Materials Science, Tel Aviv \\ University, Tel Aviv 69978, Israel \\ ${ }^{3}$ Department of Chemistry, Bar-Ilan University, Ramat-Gan 52900, Israel \\ ${ }^{4}$ ICFO - Institut de Ciencies Fotoniques, The Barcelona Institute of Science and Technology, Castelldefels \\ (Barcelona) 08860, Spain \\ ${ }^{5}$ QuTech, Delft University of Technology, P.O. Box 5046, Delft, GA 2600, The Netherlands
}

(Received 8 October 2019; revised 10 July 2020; accepted 8 September 2020; published 25 September 2020)

The standard approach to deriving fluctuation theorems fails to capture the effect of quantum correlation and coherence in the initial state of the system. Here, we overcome this difficulty and derive the heat-exchange-fluctuation theorem in the full quantum regime by showing that the energy exchange between two locally thermal states in the presence of initial quantum correlations is faithfully captured by a quasiprobability distribution. Its negativities, being associated with proofs of contextuality, are proxies of nonclassicality. We discuss the thermodynamic interpretation of negative probabilities and provide heat-flow inequalities that can only be violated in their presence. Remarkably, testing these fully quantum inequalities, at an arbitrary dimension, is no more difficult than testing traditional fluctuation theorems. We test these results on data collected in a recent experiment studying the heat transfer between two qubits and give examples for the capability of witnessing negative probabilities at higher dimensions.

DOI: 10.1103/PRXQuantum.1.010309

\section{INTRODUCTION}

Consider two systems, $C$ for "cold" and $H$ "hot," in thermal states at temperatures $T_{C}<T_{H}$. If the overall system is isolated, energy is conserved, and there are no initial correlations between $C$ and $H$, heat will flow on average from the hot to the cold body:

$$
Q \equiv Q(H \rightarrow C) \leq 0,
$$

as mandated by the second law of thermodynamics. Equation (1) can be derived for both classical and quantum systems and understood as an average over heat fluctuations that satisfy Jarzynski's exchange fluctuation theorem (XFT) [1].

The situation is less straightforward if the initial state is locally thermal but correlated, since correlations allow for temporary "back flows," i.e., $Q>0$. [2,3]. However,

\footnotetext{
*amikamlevy@gmail.com

†lostaglio@gmail.com
}

Published by the American Physical Society under the terms of the Creative Commons Attribution 4.0 International license. Further distribution of this work must maintain attribution to the author(s) and the published article's title, journal citation, and DOI. as long as the system is simply classically correlated, we can still understand these flows as emerging from averages of underlying classical fluctuations [4].

This picture breaks down in the presence of quantum correlations. In quantum theory, access to the energy of the system, which is necessary for defining fluctuations classically, can be acquired only via a measurement process that destroys the very quantum properties we wish to investigate. A striking example is given by strong heat backflows, i.e., the observation of an amount of heat flowing from $C$ to $H$ larger than $(\Delta \beta)^{-1} \log d$, where $\Delta \beta=\beta_{C}-\beta_{H}$, $\beta_{X}=1 /\left(k T_{X}\right)$, in which $k$ is Boltzmann's constant and $d$ is the local dimension. Observation of such flows implies that the underlying state is entangled [3]. If we attempt to understand this flow as originating from underlying (classical) heat fluctuations, the energy measurements required to access them destroy the entanglement and so the effect disappears. In fact, any stochastic interpretation of heat flows that reproduces the observed $Q$ faces severe no-go theorems $[5,6]$. This is the reason why the leading proposal for defining and measuring heat and work fluctuations, the "two-projective-measurement" (TPM), clearly cannot reproduce quantum effects such as strong heat back flows.

We propose that quantum heat fluctuations should be associated with a quasiprobability distribution. This arises naturally from a correction to the TPM distribution and 
can be accessed by weak measurements. We show that the associated negative "probabilities" have a clear thermodynamic interpretation as contributions to the heat flows that cannot be explained within the framework of stochastic thermodynamics. These quasiprobabilities satisfy an XFT that incorporates quantum correlations in the initial state and includes in the relevant classical limits the original XFT of Ref. [1] as well as its extension to the classically correlated systems of Ref. [4]. More importantly, our results provide heat-flow inequalities that can only be violated in the presence of negativities. These violations are a proxy for a strong form of nonclassicality known as contextuality and, remarkably, can be evaluated by means of TPM alone.

The challenges posed by the construction of a theory of heat fluctuations reproducing the observed flows are a strong indication that certain flow configurations are inherently quantum mechanical. We propose that quasiprobabilities, a tool already successfully applied in fields such as quantum optics and quantum computing, can be important diagnostic tools for exploring quantum effects in thermodynamics as our ability to measure heat flows improves.

In Sec. II, we present the problem of capturing quantum phenomena in fluctuation theorems and introduce the concept of quasiprobabilities as the solution. In Sec. III, we introduce a quasiprobability distribution for heat fluctuation and discuss its thermodynamic interpretation, its quantum nature, and the classical limit. Section IV focuses on the two-qubit system and presents heat-flow inequalities that witness negativity. We further demonstrate the result on recent experimental data. In Sec. V, we present the full quantum heat-exchange-fluctuation theorem (QXFT), and extend the results on heat-flow inequalities to arbitrarydimension systems. In Sec. VI, we show that witnessing negativity in higher-dimensional systems is possible using projective energy measurement alone and we demonstrate this on a two-qutrit system. Finally, in Sec. VII, we summarize and discuss future possible inquires.

\section{A. Comparison with previous works}

In recent years, several proposals have attempted to go beyond some of the limitations faced by TPM schemes. In Ref. [7], a modified quantum Jarzynski equality is derived, which accounts for the thermodynamic cost of the measurement. This in turn has led to approaches based on an (initial) one-time measurement scheme for closed [8] and open [9] systems, which solve experimental and conceptual difficulties related to performing the second projective measurement in the TPM scheme. Here, on the other hand, we want to confront the loss of information about quantum effects caused by the first projective measurement of the TPM scheme.

The definition of fluctuations in the quantum regime is not unique. There is in fact a zoo of such proposals that reduce to the TPM scheme in the case of initial states diagonal in the energy basis, and the first moments of which coincide with the average energy change (of the undisturbed system) (see the review in Ref. [10]). These proposals necessarily fall into one of two categories $[5,6]$ :

(1) Lack of positivity: approaches that give up the idea of assigning probabilities to fluctuations, by allowing "negative probabilities" (Margenau-Hill quasiprobability [11], full counting statistics [12-14], and consistent histories $[15,16])$. These approaches call for an interpretation of such negativities.

(2) Lack of $\sigma$-additivity: approaches based on probabilities, which give up the linearity in the density operator of the initial state (Hamilton-Jacobi framework [17], quantum Bayesian network [18], and one-time final energy measurement [19]). For example, if we consider two preparations $\rho_{\text {Heads }}$ and $\rho_{\text {Tails }}$, in these approaches the work probability for $\frac{1}{2} \rho_{\text {Heads }}+\frac{1}{2} \rho_{\text {Tails }}$ (tossing a fair coin and preparing $\rho_{\text {Heads }}$ or $\rho_{\text {Tails }}$ accordingly) is not the equal mixture of the work probability for $\rho_{\text {Heads }}$ and $\rho_{\text {Tails }}$. These approaches require an interpretation for the lack of this property, which is normally a consequence of the standard probabilistic interpretation.

Independently of the preferred alternative, given the proliferation of definitions one needs to set clear objectives for these investigations. It is not complex to come up with novel definitions of quantum fluctuations, leading to suitably complicated fluctuation theorems that differ from the standard ones due to the presence of quantum coherence. To make a proposal relevant, however, one should attempt to solve three main problems:

(a) Give an answer to the above-mentioned conceptual questions, providing a thermodynamic interpretation of the failure of properties expected in the statistical interpretation and showing how these are recovered in the classical limit.

(b) Find a definition of fluctuations leading to a notion of "quantum signature" that can be identified with a clearcut notion of nonclassicality. In other words, one should define a precise notion of classicality and show that the identified quantum signature is an operational feature that cannot be reproduced by any model satisfying that classicality property.

(c) Show that such quantum signatures are experimentally accessible without requiring a very large amount of control (such as knowledge of the initial state or the dynamics). Ideally, witnessing quantum signatures should necessitate no more control than that required by the original TPM scheme. 
What we set out to do in this work is to show that all these problems can be solved simultaneously in the context of heat fluctuations.

\section{THE PROBLEM OF QUANTUM HEAT FLUCTUATIONS}

The impasse in the definition of heat and work fluctuations can be traced back to the information-disturbance trade-off of quantum mechanics. Imagine the cold and hot systems, $C$ and $H$, initially described by a density operator $\rho_{C H}$, evolve under closed dynamics described by a unitary $U, \rho_{C H}(\tau)=U \rho_{C H} U^{\dagger}$. The average heat flow reads

$$
Q:=\operatorname{Tr}\left[\rho_{C H} H_{C}\right]-\operatorname{Tr}\left[\rho_{C H}(\tau) H_{C}\right] .
$$

In order to study fluctuations, classically we want to see this quantity as emerging from a microscopic process in which energy $q$ is exchanged with probability $p(q)$ such that $Q=\int d q p(q) q$. In this case, the system $C$ starts at some phase-space point $z_{0}$ with probability $p\left(z_{0}\right)$ and evolves deterministically to $z_{\tau}$, in which case $q=E\left(z_{0}\right)-$ $E\left(z_{\tau}\right)$, with $E(z)$ the energy at point $z$. Hence

$$
p(q)=\int d z_{0} p\left(z_{0}\right) \delta\left(q-\left(E\left(z_{0}\right)-E\left(z_{\tau}\right)\right)\right)
$$

Quantumly, however, the initial energy is generally not well defined. Noncommutativity forbids the construction of a joint probability (linear in $\rho_{C H}$ ) that correctly reproduces the energy distributions of the initial and final states [5].

This long-standing problem dates back many decades and the standard way to overcome it has been to define fluctuations by a two-point-measurement (TPM) scheme $[20,21]$. The TPM scheme involves (1) projectively measuring the energy of $C$ at the start, (2) letting the system dynamics unfold, and (3) projectively measuring the energy of $C$ again at the end. $p^{\text {TPM }}(q)$ is then defined as the probability of recording an energy difference between the outcomes of the two projective measurements equal to $q$. However, in this scheme $Q^{\mathrm{TPM}}:=\int d q p^{\mathrm{TPM}}(q) q \neq Q$, since effects due to noncommutativity are expunged by the initial invasive measurement. The widely used TPM method has the advantage that, by construction, classical fluctuation theorems hold for $p^{\mathrm{TPM}}(q)$. The drawback is that we are effectively describing the heat fluctuations of a state disturbed (decohered) by the initial energy measurement, rather than those of the original state $\rho_{C H}$. Quantum effects such as strong heat back flows provably never appear. Hence this solution cannot be satisfactory in the fully quantum regime, when $\rho_{C H}$ includes energetic coherence and quantum correlations.

\section{A. A different solution: quasiprobabilities}

We argue that the root cause of the present difficulties is the attempt to give a classical stochastic account of quantum heat fluctuations. Since quantum mechanics does not allow for the construction of joint probabilities of noncommuting quantities, a natural framework is to describe them by means of quasiprobabilities [22], i.e., real-valued functions $p(q)$ that sum to 1 but are allowed to take values outside $[0,1]$. While relatively uncommon in the thermodynamic setting (but see, e.g., Refs. $[6,11,12,14,23]$ ), the use of quasiprobabilities to probe quantum effects is widespread in other fields of quantum sciences, including quantum transport [24-26], quantum optics [27,28], quantum computing [29,30], and condensed matter [31,32]. Quasiprobabilities originated with the attempt to give a phase-space description to quantum mechanics despite noncommutativity of position and momentum. The most famous example is the Wigner function [28], which associates a quantum state with a quasiprobability distribution over positions and momenta the marginals of which correctly reproduce the statistics collected in the corresponding quantum measurements [33].

The analogy to our scenario is that, in order to recover Eq. (2), we wish to reproduce the correct quantum statistics of energy measurements performed on the initial $\rho_{C H}$ and final $\rho_{C H}(\tau)$ states. Formally, if $p^{W}{ }_{i_{C} i_{H} \rightarrow f_{C} f_{H}}$ denotes the quasiprobability of starting with energies $\left(E_{i_{C}}^{\mathrm{C}}, E_{i_{H}}^{H}\right)$ before the process $U$ and ending up in the final energies $\left(E_{f_{C}}^{\mathrm{C}}, E_{f_{H}}^{H}\right)$ afterward, then require that

$$
\begin{aligned}
& \sum_{f_{C}, f_{H}} p^{W_{i}{ }_{{ } i_{H} \rightarrow f_{C} f_{H}}}=\operatorname{Tr}\left[\rho_{C H} \Pi^{i_{C} i_{H}}\right]:=p^{i_{C} i_{H}}, \\
& \sum_{i_{C}, i_{H}} p^{W}{ }_{i_{C} i_{H} \rightarrow f_{C} f_{H}}=\operatorname{Tr}\left[\rho_{C H}(\tau) \Pi^{f_{C} f_{H}}\right]:=p^{f_{C} f_{H}}(\tau),
\end{aligned}
$$

where $\Pi^{i_{C} i_{H}} \equiv \Pi^{i_{C}} \otimes \Pi^{i_{H}}$ and $\Pi^{i_{X}}$ are energy-projection operators such that $H_{X}=\sum_{i_{X}} E_{i_{X}}^{\mathrm{X}} \Pi^{i_{X}}(X=C, H)$. Note also that $p^{i_{C} i_{H}}\left(p^{i_{C} i_{H}}(\tau)\right)$ denotes the statistics of energy measurements carried out at the beginning (end) of the experiment. The condition in Eq. (4) ensures that the fluctuations $p^{W}{ }_{i_{C} i_{H} \rightarrow f_{C} f_{H}}$ reproduce the average heat flows of Eq. (2):

$$
\sum_{i_{C}, i_{H}, f_{C}, f_{H}} p^{W}{ }_{i_{C} i_{H} \rightarrow f_{C} f_{H}}\left(E_{i_{C}}-E_{f_{C}}\right)=Q .
$$

\section{A QUASIPROBABILITY FOR QUANTUM HEAT FLUCTUATIONS}

How do we choose a quasiprobability distribution among the many possible candidates? The analogy with quantum optics suggests that different choices will be relevant in different scenarios. Nevertheless, one candidate 
stands out. Recall that the TPM distribution reads

$$
p_{i_{C} i_{H} \rightarrow f_{C} f_{H}}^{\mathrm{TPM}}:=\operatorname{Tr}\left[\Pi^{f_{C} f_{H}}(\tau) \Pi^{i_{C} i_{H}} \mathcal{D} \rho_{C H}\right],
$$

where $\Pi^{f_{C} f_{H}}(\tau):=U^{\dagger} \Pi^{f_{C} f_{H}} U$ and $\mathcal{D}$ denotes dephasing, the operation that removes all off-diagonal elements in the energy basis. Moreover, the TPM characteristic function is associated with a two-time correlation function computed on a decohered version $\mathcal{D} \rho_{C H}$ of the initial state $\rho_{C H}[20]$. This naturally suggests the reintroduction of coherence and quantum correlations by removing such dephasing:

$$
p_{i_{C} i_{H} \rightarrow f_{C} f_{H}}^{W}:=\operatorname{Re} \operatorname{Tr}\left[\Pi^{f_{C} f_{H}}(\tau) \Pi^{i_{C} i_{H}} \rho_{C H}\right],
$$

where we focus here on the real part (the imaginary part may be of independent interest [34]). This is our proposal for a quasiprobability distribution for heat fluctuations in the quantum regime.

Since every $\rho_{C H}$ can be additively decomposed as a term $\mathcal{D} \rho_{C H}$ (block-)diagonal in the energy basis plus energetic coherences $\chi_{C H}$, i.e., $\rho_{C H}=\mathcal{D} \rho_{C H}+\chi_{C H}$, corrections to the standard scheme emerge only if $\chi_{C H} \neq 0$ :

$$
p_{i_{C} i_{H} \rightarrow f_{C} f_{H}}^{W}=p_{i_{C} i_{H} \rightarrow f_{C} f_{H}}^{\mathrm{TPM}}+\operatorname{Re} \operatorname{Tr}\left[\Pi^{f_{C} f_{H}}(\tau) \Pi^{i_{C} i_{H}} \chi_{C H}\right] .
$$

The quantum correction to $p_{i_{C} i_{H} \rightarrow f_{C} f_{H}}^{\mathrm{TPM}}$ is bounded by $\left\|\chi_{C H}\right\|$, which is a coherence measure [35,36]. $p^{W}$ satisfies the marginal properties of Eq. (4) and hence it reproduces the quantum heat flow of Eq. (5). As expected, when $\chi_{C H} \neq 0$, in general $p^{W}$ does not describe a stochastic process, since it can be negative: in fact, $p_{i_{C} i_{H} \rightarrow f_{C} f_{H}}^{W} \in$ $[-1 / 8,1]$ [37]. It is worth noting that $p^{W}$ recovers the definition of a quasiprobability distribution for two noncommuting observables given by Margenau and Hill in 1961 [38] and known since the late 1930s [39]. It also takes the same form as the work quasiprobability proposed by Allahverdyan in Ref. [11], the negativities of which were, however, dismissed as an unphysical feature. Here, we present a different picture.

First, while $p^{W}$ can show negativities, it can be experimentally accessed in a probing scheme that naturally extends the TPM scheme (for more details, see Appendix A). To estimate $p^{W}$, one has to replace the first of the two projective measurements of the TPM scheme with a weak measurement, which is achieved by very weakly coupling the system to either a continuous-variable pointer or a qubit probe, which is then projectively measured. Appealingly, $p^{W}$ can hence be reconstructed in a "minimally disturbing" version of the TPM scheme.

Second, negativity is a precise signature of nonclassicality. In fact, $p_{i_{C} i_{H} \rightarrow f_{C} f_{H}}$ is proportional to a quantity inferred from weak measurements and known as the (generalized) weak value [40], first introduced by Aharonov et al. $[41,42]$ and later generalized to mixed states $[43,44]$. It then follows from the extensions [6,34] of Pusey's theorem [45] that the negativities of $p^{W}$ are witnesses of a strong form of nonclassicality known as generalized contextuality [46]. Specifically, the statistics collected by the weakmeasurement scheme probing $p^{W}$ cannot be explained by any noncontextual hidden-variable model, a claim valid even in the presence of noise [34]. This is directly related to the absence of any classical stochastic process explaining the relevant statistics [47].

Thus far, we showed that $p^{W}$ is a natural extension of the TPM scheme, both formally and experimentally; that it reproduces the heat flows of $Q$, including strong heat back flows due to initial entanglement; and that its negativity is a precise signature of strong nonclassicality. However, what is the thermodynamic significance of negativity and how does it impact the observed heat flows? We now answer these questions.

\section{A. Thermodynamic role of negativities in the heat fluctuations}

To single out the meaning of negativities, let us split the quasiprobability into positive and negative components: $p_{i_{C} i_{H} \rightarrow f_{C} f_{H}}^{W}=p_{i_{C} i_{H} \rightarrow f_{C} f_{H}}^{+}+p_{i_{C} i_{H} \rightarrow f_{C} f_{H}}^{-}$, where $p_{i_{C} i_{H} \rightarrow f_{C} f_{H}}^{ \pm}=$ $p_{i_{C} i_{H} \rightarrow f_{C} f_{H}}^{W}$ if $p_{c_{C} i_{H} \rightarrow f_{C} f_{H}}^{W}$ is positive (negative) and zero otherwise. Recall that, with our sign convention, $Q>0$ means back flow. Then, the net flow $Q$ can be decomposed into $\operatorname{direct}(H \rightarrow C)$ and back $(C \rightarrow H)$ flows:

$$
Q=Q^{\text {back }}-Q^{\text {direct }}
$$

where the "back" term includes all contributions to the flow $C \rightarrow H$ and the "direct" term includes all contributions $H \rightarrow C$ :

$$
Q^{\text {back }}=\sum_{E_{i_{C}}>E_{f_{C}}}\left(p_{i_{C} i_{H} \rightarrow f_{C} f_{H}}^{+}-p_{f_{C} f_{H} \rightarrow i_{C} i_{H}}^{-}\right) \Delta E_{i_{C} f_{C}},
$$

$$
Q^{\text {direct }}=\sum_{E_{i_{C}}>E_{f_{C}}}\left(p_{f_{C} f_{H} \rightarrow i_{C} i_{H}}^{+}-p_{i_{C} i_{H} \rightarrow f_{C} f_{H}}^{-}\right) \Delta E_{i_{C} f_{C}} .
$$

These equations give a very suggestive interpretation of the role of negative probabilities in heat flows (see Fig. 1). Since $E_{i_{C}}>E_{f_{C}}, Q^{\text {back }}$ has two positive contributions: (1) from the transition $i_{C} \rightarrow f_{C}$, which removes energy from $C$ (as expected); and (2) from the transition $f_{C} \rightarrow i_{C}$ when $p_{f_{C} f_{H} \rightarrow i_{C} i_{H}}^{-}<0$. Classically, the transition $f_{C} \rightarrow i_{C}$ would add energy to $C$, yet it contributes to the back flow from $C$ to $H$ when the correspondent quasiprobability turns negative. 

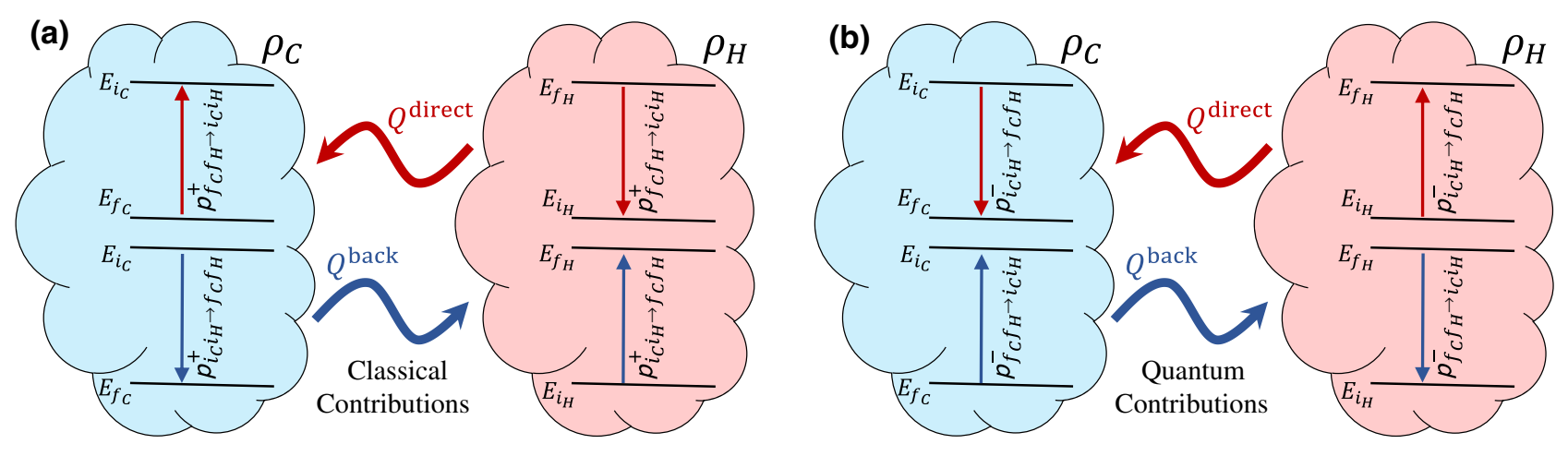

FIG. 1. A schematic illustration of the classical and quantum contributions to the overall heat flow $Q$ between a cold $(C)$ and a hot $(H)$ system. $Q$ is an average of direct $(H \rightarrow C)$ and back flows $(C \rightarrow H)$. (a) The classical contributions (positive-probability events) correspond to standard contributions, where the loss of energy from $H$ contributes to the flow toward $C$ and vice versa. (b) The quantum contributions (negative-probability events) effectively act as contributions in which the loss of energy from $H$ contributes to the flow $(C \rightarrow H)$.

Symmetrically, a transition taking energy away from $C$ can contribute to the direct flow, when $p_{i_{C} i_{H} \rightarrow f_{C} f_{H}}^{-}<0$. These counterintuitive heat-flow contributions signal the breakdown of a stochastic description and the onset of quantum effects, as depicted in Fig. 1.

In particular, we can understand strong flows as the result of negative probabilities, which are destroyed by the measurement stage of the TPM scheme (a projective energy measurement on $C$ and $H$ forces $p^{W}>0$ ). Furthermore, Eq. (10) suggests that negativities may lead to strong direct flows as well.

It is important to bear in mind that negativity will not always result in strong heat flows. Since only $Q=Q^{\text {back }}-$ $Q^{\text {direct }}$ is observed, negativities from $Q^{\text {back }}$ and $Q^{\text {direct }}$ must not cancel each other if we are going to observe an overall effect in the heat flow.

\section{B. Classical limit}

When discussing quantum effects in heat or work fluctuations, it is important to distinguish between two different forms:

1. Quantumness due to correlations or entanglement in the initial state, such as that responsible for strong heat back flows.

2. Quantumness due to the superpositions created by $U$ during the dynamics.

The first form of nonclassicality is the one that prevents a straightforward description of quantum heat exchanges by means of a classical stochastic process. Classicality in the first sense emerges in the expected regimes. The typical mechanism is decoherence. If $C$ and $H$ are not protected from the external environment, superpositions of different energy states are quickly suppressed $\left(\chi_{C H} \rightarrow 0\right)$ and so
$p^{W} \rightarrow p^{\mathrm{TPM}} \geq 0$, i.e., negativity disappears. Decoherence may be also due to the measurement process, which is the mechanism that introduces classicality in the TPM scheme.

Another mechanism relevant at mesoscopic scales is coarse graining. Suppose that one can only access the heat exchanges between two clusters, each containing $N$ particles, but one cannot keep track of the process at the level of the individual constituents. We idealize the situation via an average Hamiltonian $H_{C H} \approx \sum_{i=1}^{N}\left(H_{C_{i}}+\right.$ $\left.H_{H_{i}}\right) / N$ and dynamics $U \approx \otimes_{i=1}^{N} U_{C_{i} H_{i}}$. Then, whatever the initial state $\rho_{C H}$ is, $p^{W} \approx p^{\mathrm{TPM}}+O(1 / N)$ [48]. The ratio between fluctuations and average flows, on the other hand, is suppressed as $O(1 / \sqrt{N})$. There is hence an intermediate "mesoscopic" regime in which quantum effects are irrelevant but fluctuations are not yet negligible.

The second form of nonclassicality has been studied in Ref. [49]. In this study, it has been shown that for an initially uncorrelated bipartite system, energy cannot flow without generation of quantum correlations in the form of discord during the unitary process. This form of quantumness is the one responsible for the differences between the classical stochastic process predicted by the TPM scheme and the one obtained within a fully classical description (e.g., a classical-mechanics model), whenever the latter is well defined. Note that since we recover the TPM probability in the regimes discussed above, we can exploit previous results on the emergence of this second form of classicality when the superpositions created by $U$ during the dynamics can be neglected [50].

We conclude that our definition of heat fluctuations appears to provide a clear path to the emergence of classicality, from negative quasiprobability all the way up to a classical-mechanical model, passing through the standard TPM scheme that plays the role of a "quasiclassical" description. 


\section{HEAT FLOWS WITNESSING NEGATIVITIES IN A TWO-QUBIT SYSTEM}

By attempting to derive a stochastic interpretation of quantum fluctuations, we arrive at a picture in which quantum effects are incorporated as nonclassical features in the heat exchange, such as contributions to the energy flow from $C$ to $H$ associated with transitions to higher levels in $C$. From a practical perspective, however, the next step is to ask if these nonclassical features can be directly related to observable effects and if these effects can be accessed in realistic experiments. We answer both questions in the positive.

We start with the archetypal (and fully solvable) idealized two-qubit scenario. Since $U$ injects no work, nontrivial energy exchanges can only happen if the two qubits are resonant.

Inequality 1. Let $\rho_{C H}$ be a two-qubit system with thermal marginals $\left(\beta_{C} \neq \beta_{H}\right), H_{C}=H_{H}=E|1\rangle\langle 1|$ and $U a$ unitary injecting no work, $\left[U, H_{C}+H_{H}\right]=0$. If $p^{W}$ is non-negative,

$$
|Q| \leq \frac{2+e^{\beta_{H} E}+e^{\beta_{C} E}}{e^{\beta_{C} E}-e^{\beta_{H} E}}\left|Q^{\mathrm{TPM}}\right| .
$$

See Appendix B 1 for the proof. This "heat-flow inequality" shows that strong enough direct or back flows of heat can only occur when negativity is at play in $p^{W}$. Note that to violate the bound of Eq. (11), we necessarily witness $|Q|>\left|Q^{\mathrm{TPM}}\right|$, i.e., a direct or inverse flow bigger than the corresponding flow observed in the TPM scheme. While violations of Eq. (1), reported in Ref. [51], can occur in a purely classical setup, due to initial correlations in the energetic degrees of freedom, violations of Inequality 1 imply negativity, which is a proxy for genuinely quantum effects.

\section{A. Experimental verification}

Next, we analyze the violations of Inequality 1 . In recent NMR experimental setups [51,52], the heat back flow between two local-thermal qubits with initial correlations has been measured. Here, we focus on the data of Ref. [51] and show that, while other general approaches (e.g., Ref. [3]) fail to witness quantum effects for this setup, Inequality 1 is violated.

In the experiment, the heat-back-flow $Q$ between two nuclear spin $1 / 2$, prepared in an initial locally thermal state, has been measured. We denote the Pauli matrices by $\sigma_{i}$ and $i=x, y, z$. The initial Hamiltonian is $H_{H C}=H_{H}+$ $H_{C}$, in which $H_{C(H)}=h v\left(1-\sigma_{z}^{C(H)}\right)$ and $h$ is Planck's constant. A locally thermal state is prepared with the form

$$
\rho_{H C}=\rho_{H} \otimes \rho_{C}+\gamma|01\rangle\left\langle 10\left|+\gamma^{*}\right| 10\right\rangle\langle 01|,
$$

where $\rho_{H(C)}=e^{-\beta_{H(C)} H_{H(C)}} / \operatorname{Tr}\left[e^{-\beta_{H(C)} H_{H(C)}}\right]$. The unitless rescaled inverse temperatures reported in the experiment are $\beta_{H}=0.9618$ and $\beta_{C}=1.13$, with the gap $v=1 \mathrm{kHz}$. In the correlated scenario, $\gamma=-0.19$, whereas in the uncorrelated scenario $\gamma=0$.

During the process, the nuclear spins are coupled via the interaction Hamiltonian $H_{\text {int }}=J \pi \hbar / 2\left(\sigma_{x}^{H} \sigma_{y}^{C}-\sigma_{y}^{H} \sigma_{x}^{C}\right)$, where $J=215.1 \mathrm{~Hz}$. Since $\left[H_{\text {int }}, H_{H C}\right]=0$, this generates an energy-preserving unitary. A simple yet important observation is the following: the measurement of the heat exchange when $\gamma=0$ corresponds to the measurement of $Q^{\mathrm{TPM}}$ for the case $\gamma=-0.19$. This follows from the fact that the initial energy measurement on $C$ and $H$ (the first step of the TPM protocol) has the effect of setting $\gamma=0$. Due to this observation, the data collected in the experiment suffice to test Inequality 1.

In Fig. 2, we plot the heat transfer between qubits $C$ and $H$ for various interaction times. The green line corresponds to the heat flow when the qubits are initially quantum correlated, whereas the orange line corresponds to initially uncorrelated qubits. Equivalently, this corresponds to the measurements of $Q$ (green) and $Q^{\mathrm{TPM}}$ (orange). $Q>0$ corresponds to back flow from $C$ to $H$, whereas $Q<0$ corresponds to direct flow from $H$ to $C$. The shaded areas indicate the interaction times in which negativity in heattransfer quasiprobability is present. The dark blue shaded area indicates the interaction times for which Inequality 1 successfully witnesses negativity for the relevant experimental parameters.

While in the experiment only the back-flow regime has been explored, which is sufficient for detecting negativity, here we theoretically extend the interaction time to the regime in which direct flow is observed. We see that in this regime also, the measurement of the heat flows would allow us to detect the negativity of the quasiprobability, showing that quantum effects are not only present in back flows. We further extend the investigation of Inequality 1 beyond the specific parameters of Ref. [51] (see Fig. 3). We

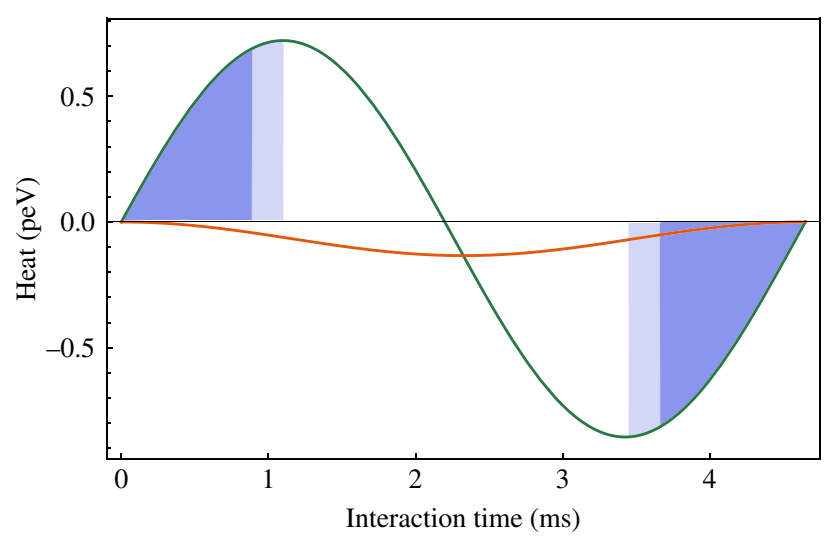

FIG. 2. The heat transfer $Q$ and $Q^{\mathrm{TPM}}$ (green and orange curves, respectively) for the experiment in Ref. [51]. The shaded areas indicate the regime of negative probabilities. The dark blue shaded area indicates the violation of Inequality 1. 


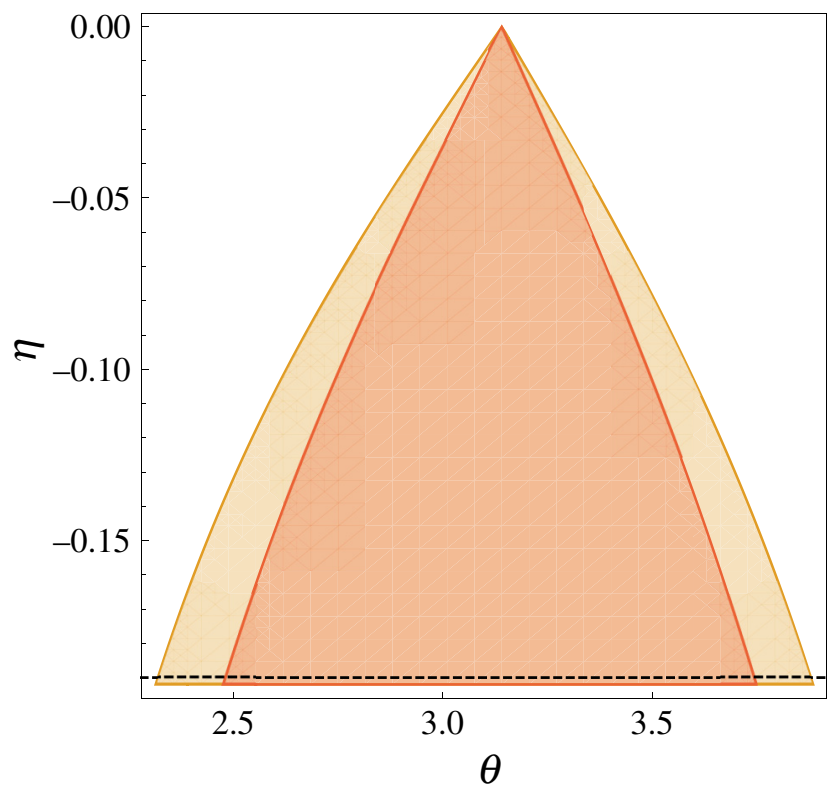

FIG. 3. The region of negative probabilities (large trianguloid) and violations of Inequality 1 (darker trianguloid) in the parameter space $(\theta, \eta) . \theta$ characterizes the unitary evolution (the rotation in the $|01\rangle,|10\rangle$ subspace) and $\eta$ the initial energetic superposition between $|01\rangle,|10\rangle$. In Appendix B, we show that for fixed $\beta_{C}, \beta_{H}$ and a simple reparametrization, the parameter space is restricted to $\left(\theta, \eta, P_{00}\right)$, where $P_{00}$ is the population in the state $|00\rangle$. In the experiment reported in Ref. [51], $\beta_{C}=1.13$, $\beta_{H}=0.962, P_{00}=0.547$, and $\eta=-0.19$, which is indicated by the dashed line at the bottom of the figure.

conclude that negativity can be detected for a wide range of parameters in accessible experiments.

Using the experimental parameters of Ref. [51], the smallest eigenvalue of the partial transpose $\rho_{C H}^{T_{H}}$ of $\rho_{C H}$ is approximately 0.0014 ; hence $\rho_{C H}^{T_{H}} \geq 0$. By the PeresHorodecki criterion, $\rho_{C H}$ is separable. This shows that the heat fluctuations can have negativities, as detected by Inequality 1 , even when $\rho_{C H}$ is not entangled.

We mention in passing a nontrivial result that holds specifically for two-qubit systems: the existence of quantum correlations in the energy degrees of freedom is necessary for witnessing a back flow for appropriate energypreserving dynamics (see Appendix B). This is the reason why the orange curve in Fig. 2 is all below the $x$ axis.

\section{B. Nonideal heat exchange}

While it is commonplace to assume that $U$ injects an amount of work into the system that can be neglected in calculations, strictly speaking, Inequality 1 holds only in an ideal case. Next, we extend Inequality 1 to realistic heatexchange scenarios, in which (1) the energy levels are not resonant and (2) the dynamics inject some work.
Inequality 2. Let $\rho_{C H}$ be a two-qubit system with thermal marginals $\left(\beta_{C} \neq \beta_{H}\right), \quad H_{C}=E_{C}|1\rangle\left\langle 1\left|, \quad H_{H}=E_{H}\right| 1\right\rangle\langle 1|$ and let $U$ denote unitary dynamics. Suppose that

1. $U$ is close to an energy-preserving unitary $\tilde{U}$ : $\|U-\tilde{U}\| \leq \epsilon$.

2. The gap is not too wide: $\Delta:=\left|E_{C}-E_{H}\right| / 2 \bar{E}<$ $(1-R)(1+R)$, where $\bar{E}:=\left(E_{C}+E_{H}\right) / 2$ is the average energy and $R=\left(1+e^{\beta_{H} E_{H}}\right) /\left(1+e^{\beta_{C} E_{C}}\right)$.

3. The energy flow is large enough that we can distinguish a heat contribution: $|Q|>2 \epsilon \bar{E}$.

If $p^{W}$ is non-negative,

$|Q| \leq \frac{1+R+\Delta(1+R)}{1-R-\Delta(1-R)}\left|Q^{\mathrm{TPM}}\right|+4 \epsilon \bar{E} \frac{2+\Delta(1+R)}{1-R-\Delta(1-R)}$.

When $\epsilon \rightarrow 0$ and $\Delta \rightarrow 0$, we recover Inequality 1 . The above inequality is suitable to be applied in realistic experimental scenarios. Note that the condition of unitarity is not crucial and that Inequality 2 can be extended to quantum channels. The proof of this inequality, stronger bounds, and a case study are presented in Appendix B 2. This shows that analog heat-flow inequalities can be derived beyond the ideal regime.

\section{A QUANTUM-EXCHANGE-FLUCTUATION THEOREM}

The above heat-flow inequality is limited to two-qubit systems. We now derive a heat-flow inequality valid for arbitrary finite-dimensional systems. This is obtained through the derivation of a QXFT in the spirit of Ref. [11, 53].

Denote by $\Delta I:=I_{f_{C} f_{H}}-I_{i_{C} i_{H}}$, with

$$
I_{i_{C} i_{H}}=\log \frac{\operatorname{Tr}\left[\Pi^{i_{C} i_{H}} \rho_{C H}\right]}{\operatorname{Tr}\left[\Pi^{i_{C}} \rho_{C}\right] \operatorname{Tr}\left[\Pi^{i_{H}} \rho_{H}\right]},
$$

the elements of the classical mutual information. Assuming that $E_{i_{C}}-E_{f_{C}} \approx E_{f_{H}}-E_{i_{H}}$ for all nonzero $p^{W}{ }_{i_{C} i_{H} \rightarrow f_{C} f_{H}}$, we obtain the XFT in the full quantum regime

$$
\left\langle e^{\Delta I+\Delta \beta \Delta E_{i} C_{C}}\right\rangle_{W}=1+\bar{\chi} .
$$

The right-hand side of Eq. (15) consists of 1, the contribution from the populations, and $\bar{\chi}$, involving contributions from the coherence terms in the energy eigenbasis

$$
\bar{\chi}=\sum_{l, k \neq m} \frac{\rho_{l l}}{\rho_{k k}} \operatorname{Re}\left\{\rho_{k m}\langle l|U| k\rangle\left\langle m\left|U^{\dagger}\right| l\right\rangle\right\}
$$

which are absent in the TPM scheme. Note the following: 
1. If $\rho_{C H}$ is classically correlated in the energy basis, $\bar{\chi}=0$, recovering the main result of Ref. [4].

2. If, furthermore, $\rho_{C H}$ is uncorrelated, $\Delta I=0$ and we recover Jarzynski's original result [1].

Equation (15) is thus the generalization of the XFT to the full quantum regime. The proof of the quantum XFT is provided in Appendix C.

\section{A. Witnessing negativity in an arbitrary dimension}

Using the quantum XFT, we derive a general inequality witnessing negativity in any dimension.

Inequality 3. Let $\rho_{C H}$ be an arbitrary finite-dimensional system with thermal marginals $\left(\beta_{C} \neq \beta_{H}\right)$ and let $U$ be a unitary that injects no work $\left(E_{i_{C}}-E_{f_{C}}=E_{f_{H}}-E_{i_{H}}\right)$. If $p^{W}$ is non-negative,

$$
Q \leq-\frac{\langle\Delta I\rangle_{\mathrm{W}}}{\Delta \beta}+\frac{\log (1+\bar{\chi})}{\Delta \beta}
$$

This result can be derived by applying Jensen's inequality to Eq. (15). $\langle\Delta I\rangle_{W}$ in Eq. (17) is a classical mutual informationlike term satisfying $\langle\Delta I\rangle_{\mathrm{W}}=\langle\Delta I\rangle_{\mathrm{TPM}}$ if the initial state only has classical correlations in the energy basis and $\langle\Delta I\rangle_{W}=0$ in the absence of initial quantum and classical correlations [54]. Violations of the inequality imply that some quasiprobabilities are necessarily negative. Note that the inequality becomes trivial if no initial coherence is present $\left(\chi_{C H}=0\right)$, as expected. In Appendix $\mathrm{C}$, we provide a second heat-flow inequality that is valid for general finite-dimensional systems.

For finite-dimensional systems with equal Hamiltonians and nondegenerate energy gaps, we prove in Appendix $\mathrm{C}$ an additional generic property of negativity: if all quasiprobabilities that contribute to direct or back flow are negative, then the flows are necessarily strong, i.e., $|Q|>\left|Q^{T P M}\right|$.

\section{B. Nonideal heat exchange}

Inequality 3 can be generalized to situations in which the injected work cannot be neglected. If, for all nonzero $p^{W} i_{i_{C} i_{H} \rightarrow f_{C} f_{H}}$, one has $\left|E_{i_{C}}-E_{f_{C}}-\left(E_{f_{H}}-E_{i_{H}}\right)\right| \leq$ $\epsilon$, almost the same derivation allows us to generalize Eq. (17) to

$p^{W}$ non-negative $\Rightarrow Q \leq-\frac{\langle\Delta I\rangle_{\mathrm{W}}}{\Delta \beta}+\frac{\log (1+\bar{\chi})}{\Delta \beta}-\frac{\beta_{H} \epsilon}{\Delta \beta}$.

A symmetric inequality can be derived for the energy flowing into $C$.

\section{Case study: heat exchange between two qutrits}

In this section, we investigate the heat flows between two qutrits and show that Inequality 3 can witness negativity in these higher-dimensional systems as well. The Hamiltonian of the qutrits is assumed to take the form $H_{C}=H_{H}=\sum_{n=0}^{2} E_{n}|n\rangle\langle n|$, where $E_{0}=0$ and there is no degeneracy of the energy gaps (the "Bohr spectrum"). The state $\rho_{C H}=\sum_{i, j=0}^{8} \rho_{i j}|i\rangle\langle j|$ can be expressed by the elements $\rho_{i j}=\rho_{j i}^{*}=\eta_{i j} e^{-i \xi_{i j}} \sqrt{\rho_{i} \rho_{j}} \forall i \neq j$, the population $\rho_{i} \equiv \rho_{i i}$, and the parameters $\eta_{i j} \in[0,1]$ and $\xi_{i j} \in \mathbb{R}$. Here, we use the natural labeling $(00,01,02,10,11,12, \ldots) \equiv$ $(0,1,2,3,4,5, \ldots)$.

By imposing the constraint that the marginal states $\operatorname{Tr}_{C}\left[\rho_{C H}\right]$ and $\operatorname{Tr}_{H}\left[\rho_{C H}\right]$ are thermal, and following the same reasoning as in the two-qubit scenario, heat flows between two qutrits are determined by: the six parameters $\eta_{i j}, \quad \xi_{i j}$ for $(i, j)=\{(01,10) \equiv(1,3),(02,20) \equiv$ $(2,6),(12,21) \equiv(5,7)\}$; and four undetermined populations $\rho_{i}$ that must comply with the non-negativity of $\rho_{C H}$. Furthermore, all possible heat flows achievable by energypreserving unitaries can be realized by $U=\bigoplus_{i=1}^{3} U^{(i)}$, where $U^{(i)}$ are real $2 d$ rotations within the three abovementioned manifolds, fixed by three angles $\theta_{01}, \theta_{02}$, and $\theta_{12}$. In Appendix C, we provide the explicit structure of the state $\rho_{C H}$ and the energy-preserving unitary. In Fig. 4, we plot the regions in parameter space $\theta_{01}$ and $\theta_{02}$ in

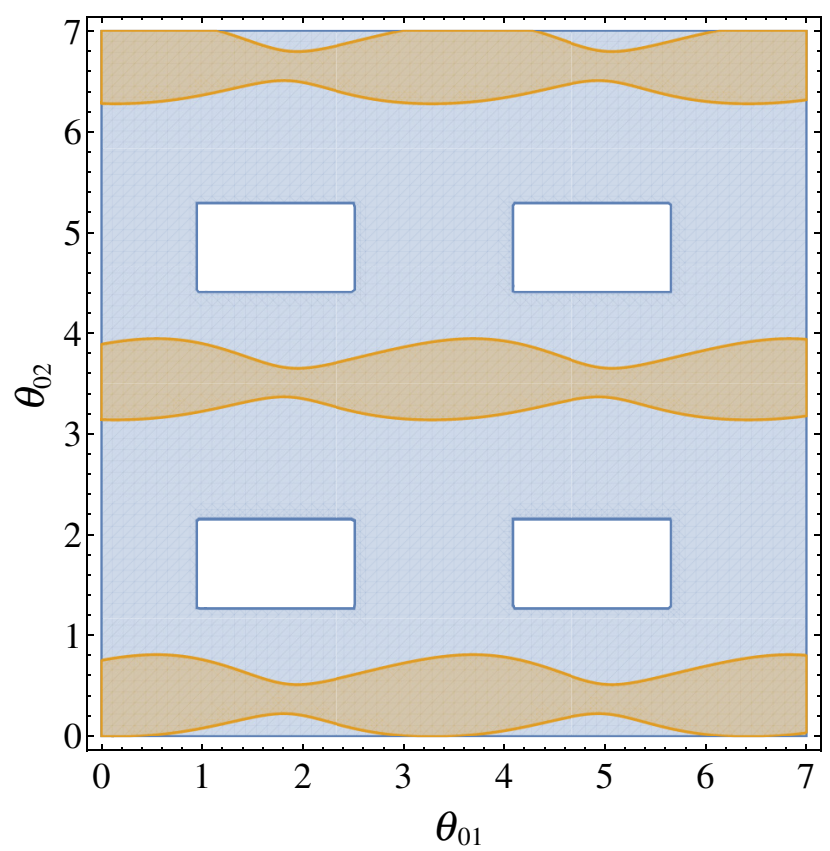

FIG. 4. The negativity of the quasiprobabilities (all shaded areas) and the violation of Inequality 4 (yellow shaded area) for different interaction protocols determined by $\theta_{01}$ and $\theta_{02}$. Parameters: $\beta_{H}=0.3, \beta_{C}=1.3, \theta_{12}=\theta_{02}, E_{1}=1, E_{2}=1.15, \xi_{i j}=$ $0, \eta_{i j}=1$ for $(i, j)=\{(1,3),(2,6),(5,7)\}, \rho_{0}=0.3, \rho_{5}=0.03$, $\rho_{7}=0.07$, and $\rho_{8}=0.06$. 
which these probabilities turn negative (all shaded areas). The yellow shaded area indicates the regions in which Inequality 3 is being violated. This clearly shows that our method also allows us to detect negativities at higher dimensions. A more detailed analysis and a straightforward generalization to an arbitrary dimension are presented in Appendix C.

\section{WITNESSING NEGATIVITY WITH PROJECTIVE ENERGY MEASUREMENTS IN AN ARBITRARY DIMENSION}

The testing of violations of Eq. (17) is a way to probe the fully quantum regime in arbitrary finite-dimensional systems. However, it has a significant drawback of being experimentally very demanding, as $\bar{\chi}$ depends on all the entries in the initial state, as well as the details of the dynamics. Standard fluctuation-theorem experiments, on the other hand, involve only projective energy measurements. The drawback of these is the inability to probe quantum effects due to initial quantum correlations and energy coherence. Can our method be adapted to obtain a scheme satisfying both desiderata, that is, (1) probing the fully quantum regime and (2) using only the experimental capabilities required by the traditional TPM quantum fluctuation theorems? Here, we answer this question in the positive.

We consider two $d$-dimensional systems and for simplicity we assume that there are no matching gaps in their energy spectra (formally, the "nondegenerate Bohr spectrum"). The most general energy-preserving unitary is then a direct product of two-dimensional rotations. This allows us to derive the following result (see Appendix $\mathrm{C}$ for the proof).

Inequality 4. Consider two arbitrary finite-dimensional systems with nondegenerate Bohr spectra undergoing an energy-preserving unitary. If $p^{W}$ is non-negative, then

$$
Q \in\left[Q^{\mathrm{TPM}}-2 \lambda_{-}, Q^{\mathrm{TPM}}+2 \lambda_{+}\right]
$$

where

$$
\begin{aligned}
& \lambda_{-}=\sum_{E_{i_{C}}>E_{f_{C}}} p^{\mathrm{TPM}}{ }_{i_{C} i_{H} \rightarrow f_{C} f_{H}} \Delta E_{i_{C} f_{C}}, \\
& \lambda_{+}=\sum_{E_{i_{C}}<E_{f_{C}}} p^{\mathrm{TPM}}{ }_{{ }_{i} i_{H} \rightarrow f_{C} f_{H}} \Delta E_{f_{C} i_{C}} .
\end{aligned}
$$

and $\Delta E_{n m}:=E_{n}-E_{m}$.

Remarkably, to confirm a violation of Eq. (19) and witness negativity, it suffices to have the experimental capability of performing projective energy measurements. In fact, $\lambda_{-}$and $\lambda_{+}$and $Q^{\mathrm{TPM}}$ can all be inferred from the data already required to test standard TPM fluctuation theorems. To infer $Q$, one can prepare $N$ samples of the initial state and use half to estimate the average energy before the dynamics and half to estimate the average energy after the dynamics. The difference between the two is the heat $Q$. In other words, if one can protect the initial state from decoherence, negativity can be witnessed in any of the platforms used to test TPM fluctuation theorems. It is worth noting that Inequality 4 does not require local thermality. In other words, it can also be tested measuring energy exchange between two quantum systems in a generic state.

To illustrate this result, we consider the two-qutrits case study of Sec. VC. Figure 5 presents the negativity of the quasiprobability related to heat exchange (all shaded areas) and the violation of the Inequality 4 (green and yellow areas) for different interaction protocols determined by the unitary angles $\theta_{01}$ and $\theta_{02}$. The different insets represent different initial states, determined by the parameter $\eta=\eta_{i j}$ for $(i, j)=\{(1,3),(2,6),(5,7)\}$ that quantifies the amount of initial quantum correlations between the states. The yellow area represents violation of the lower bound
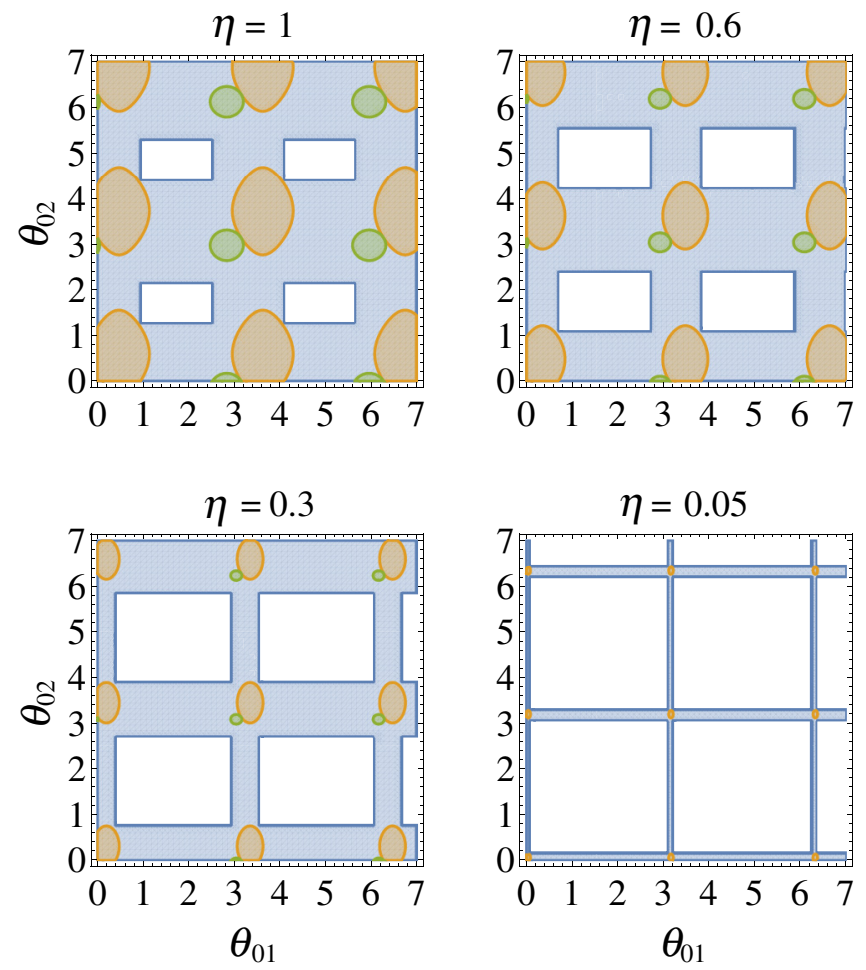

FIG. 5. The negativity of the quasiprobabilities (all shaded areas) and the violation of Inequality 4 (yellow and green shaded area) for different interaction protocols determined by $\theta_{01}$ and $\theta_{02}$. The insets shows different initial states, determined by the parameter $\eta=\eta_{i j}$ for $(i, j)=\{(1,3),(2,6),(5,7)\}$ Parameters: $\beta_{H}=$ $0.3, \beta_{C}=1.3, \theta_{12}=\theta_{02}, E_{1}=1, E_{2}=1.15, \xi_{i j}=0, \rho_{0}=0.3$, $\rho_{5}=0.03, \rho_{7}=0.07$, and $\rho_{8}=0.06$. 
of Inequality 4 and corresponds to negativity in the direct flow as illustrated in the quantum contributions of Fig. 1 (upper-right process, red arrows). The green area, on the other hand, represents violations of the upper bound of Inequality 4, which correspond to negativity in the back flow as illustrated in the quantum contributions of Fig. 1 (lower-right process, blue arrows). The upper-left inset of Fig. (5) with $\eta=1$ corresponds to the parameters used for Fig. 4. This indicates that Inequalities 3 and 4 can witness negativity in different regimes. As already remarked, however, the testing of Inequality 4 is much simpler.

\section{OUTLOOK}

Recent no-go results $[5,6]$ strongly suggest that, if we are to probe the fully quantum thermodynamic regime, due to noncommutativity we need to renounce a straightforward statistical interpretation of microscopic fluctuation processes. Moving in this direction, here we introduce a natural quasiprobability for heat fluctuations the negativity of which captures nonclassical effects in the strong form of contextuality. This formally implies the impossibility of describing the weak-measurement scheme probing $p^{W}$ as the result of any underlying classical stochastic process. Differently from previous approaches, we show that negativities in the heat fluctuations have a thermodynamic interpretation and that certain heat flows between two locally thermal states at different temperatures can only happen in their presence. To prove the latter, we develop families of heat-flow inequalities that can only be violated in the presence of negativities. We use these tools to witness nonclassicality by analyzing data collected in a recent two-qubit experiment. We also present heat-flow inequalities for generic finite-dimensional systems, the testing of which only requires the control already needed to test standard TPM fluctuation theorems, with the advantage that one is testing the fully quantum regime.

Moving forward, we propose that a quasiprobability approach to fluctuations can advance our understanding of the thermodynamic behavior of realistic devices and the study of heat flows in more complex networks. Classically unachievable heat-flow configurations can also be used as a witness of nonclassicality in thermal machines and could unlock performance boosts in thermodynamic protocols. We also expect that the same ideas as developed here can be applied to work fluctuations as well. Building on the seminal paper of Allahverdyan [11], this may provide the most direct experimental test of the framework developed here.

More broadly, this work also opens up the possibility of applying to nonequilibrium thermodynamics the extensive technical tools based on quasiprobability representations developed to identify quantum effects and advantages in quantum optics, quantum foundations, quantum computing, and condensed-matter physics.

\section{ACKNOWLEDGMENTS}

We thank David Jennings and Karen Hovhannisyan for helpful discussions. M.L. thanks Eran Rabani for the kind hospitality during a visit to University of California, Berkeley, where part of this work was completed. M.L. acknowledges financial support from the European Union's Marie Sklodowska-Curie individual Fellowships (Grants No. H2020-MSCA-IF-2017 and No. GA794842), the Spanish Ministerio de Econom y Competitividad (Ministry of Economy and Competitiveness, MINECO) (Severo Ochoa Grant No. SEV-2015-0522 and Project No. QIBEQI FIS2016-80773-P), the Fundacio Cellex and the Generalitat de Catalunya (Centres de Recerca de Catalunya, CERCA Program and SGR 1381). This work is part of the "Photonics at Thermodynamic Limits" Energy Frontier Research Center funded by the U.S. Department of Energy, Office of Science, Office of Basic Energy Sciences under Award No. DE-SC0019140.

A.L. and M.L. contributed equally to this work.

\section{APPENDIX A: ESTIMATING THE MARGENAU-HILL QUASIPROBABILITY}

\section{a. Traditional scheme}

The scheme for measuring $p^{W}{ }_{i_{C} i_{H} \rightarrow f_{C} f_{H}}$ consists of a weak measurement at the start of the protocol and a projective measurement at the end. Specifically, define a family of measurement schemes where at the start the system projectors $\Pi^{i_{C}{ }^{i} H}$ are coupled for a unit time to the momentum $P$ of a one-dimensional pointer device through the interaction Hamiltonian $\Pi^{i} C^{i} H \otimes P$. The pointer is initially in a pure state $\left(\pi s^{2}\right)^{-1 / 4} \int d x e^{-x^{2} / 2 s^{2}}|x\rangle$. Then the dynamics, represented by some unitary $U$, take place on $\mathrm{CH}$. At the end, a final projective energy measurement is performed on $\mathrm{CH}$ and outcome $\left(f_{C}, f_{H}\right)$ is observed with probability $q_{f_{C} f_{H}}$.

One can then verify that the expected position of the pointer given that some energies $\left(f_{C}, f_{H}\right)$ are observed in the final energy measurement, denoted by $\langle X\rangle_{f_{C} f_{H}}$, can be directly related to $p^{W}{ }_{i_{C} i_{H} \rightarrow f_{C} f_{H}}$ in the weak-measurement limit $s \rightarrow \infty$ (large initial spread of the pointer):

$$
\langle X\rangle_{\mid f_{C} f_{H}} q_{f_{C} f_{H}} \stackrel{s \rightarrow \infty}{\longrightarrow} p^{W}{ }_{i_{C} i_{H} \rightarrow f_{C} f_{H}} .
$$

The same expression gives $p^{\mathrm{TPM}}{ }_{i_{C} i_{H} \rightarrow f_{C} f_{H}}$ if $s \rightarrow 0$ (very sharp pointer). For a derivation see, for example, Appendix $\mathrm{B}$ of Ref. [6]. In this sense, the protocol probing $p^{W}{ }_{i_{C} i_{H} \rightarrow f_{C} f_{H}}$ can be understood as a "minimally invasive" version of the standard TPM scheme.

\section{b. Qubit probe}

An alternative scheme to estimate $p^{W}{ }_{i_{C} i_{H} \rightarrow f_{C} f_{H}}$ uses only a qubit pointer rather than a continuous system $[34,56]$ 
and, for completeness, is briefly discussed here. Take a qubit ancilla in a state $\left|\psi_{\epsilon}\right\rangle=\cos \epsilon|0\rangle-\sin \epsilon|1\rangle$. Couple the system and the ancilla through the unitary $V=$ $\Pi^{i_{C} i_{H} \perp} \otimes \mathbb{I}+\Pi^{i^{i} i_{H}} \otimes \sigma_{z}$ (generated by the Hamiltonian $H_{\text {int }}=\Pi^{i{ }^{i} i_{H}} \otimes|1\rangle\langle 1|$ by $V=e^{-i g H_{\text {int }} t}$, setting $\left.t=\pi / g\right)$. The ancilla is then measured in the $| \pm\rangle=(|0\rangle \pm|1\rangle) / \sqrt{2}$ basis. Denoting the two outcomes by \pm , the corresponding Kraus operators on the system are

$$
M_{ \pm}=\left\langle \pm|V| \psi_{\epsilon}\right\rangle=\frac{\cos \epsilon}{\sqrt{2}} \mathbb{I} \pm \frac{\sin \epsilon}{\sqrt{2}}\left(\Pi^{i_{C} i_{H}}-\Pi^{i_{C} i_{H} \perp}\right)
$$

with the correspondent positive-operator-valued measurement (POVM) on the system being

$$
E_{ \pm}=M_{ \pm}^{\dagger} M_{ \pm}=(1 \mp \sin 2 \epsilon) \frac{\mathbb{I}}{2} \pm \sin 2 \epsilon \Pi^{i_{C} i_{H}}
$$

Denote the joint probability of observing outcome + on the pointer and outcome $\left(f_{C}, f_{H}\right)$ on the system by $q_{f_{C} f_{H},+}(\epsilon)$. Also define, with obvious notation, $q_{f_{C} f_{H},-}(\epsilon)$. Then, if $\Delta q_{f_{C} f_{H}}(\epsilon):=q_{f_{C} f_{H},+}(\epsilon)-q_{f_{C} f_{H},-}(\epsilon)$,

$$
\begin{aligned}
\Delta q_{f_{C} f_{H}}(\epsilon) & \\
= & \operatorname{Tr}\left[\Pi^{f_{C} f_{H}}(\tau) \otimes|+\rangle\langle+| V\left(\rho_{C H} \otimes\left|\psi_{\epsilon}\right\rangle\left\langle\psi_{\epsilon}\right|\right) V^{\dagger}\right] \\
& -\operatorname{Tr}\left[\Pi^{f_{C} f_{H}}(\tau) \otimes|-\rangle\langle-| V\left(\rho_{C H} \otimes\left|\psi_{\epsilon}\right\rangle\left\langle\psi_{\epsilon}\right|\right) V^{\dagger}\right] \\
= & \sin 2 \epsilon\left(2 p^{W}{ }_{i_{C}{ } i_{H} \rightarrow f_{C} f_{H}}-p_{f_{C} f_{H}}\right),
\end{aligned}
$$

where $\Pi^{f_{C} f_{H}}(\tau)=U^{\dagger} \Pi^{f_{C} f_{H}} U$ and $p_{f_{C} f_{H}}=\operatorname{Tr}\left[\Pi^{f_{C} f_{H}}(\tau) \rho_{C H}\right]$ is the probability of observing energy outcomes $\left(f_{C} f_{H}\right)$ if no measurement scheme is performed $(V=\mathbb{I})$. Clearly,

$$
p^{W}{ }_{i_{C} i_{H} \rightarrow f_{C} f_{H}}=\frac{\Delta q_{f_{C} f_{H}}(\epsilon)}{2 \sin 2 \epsilon}+\frac{p_{f_{C} f_{H}}}{2} .
$$

This gives a way of reconstructing $p^{W} i_{i_{C} i_{H} \rightarrow f_{C} f_{H}}$ from the joint statistics on the pointer and the system in the abovementioned measurement scheme, together with the probabilities $p_{f_{C} f_{H}}$ that can be inferred from a second experiment where no measurement scheme is applied. Note that, given the knowledge of $\epsilon$ from the initialization of the ancilla, one can reconstruct $p^{W}{ }_{i_{C} i_{H} \rightarrow f_{C} f_{H}}$ without taking the limit $\epsilon \rightarrow 0$.

\section{APPENDIX B: HEAT FLOW BETWEEN TWO QUBITS}

\section{a. Reduction of the parameters}

The most general two-qubit unitary satisfying energy conservation takes the form

$$
U=\left(\begin{array}{cccc}
1 & 0 & 0 & 0 \\
0 & e^{i(\kappa+\lambda)} \cos \theta & -e^{i(\kappa-\phi)} \sin \theta & 0 \\
0 & e^{i(\kappa+\phi)} \sin \theta & e^{i(\kappa-\lambda)} \cos \theta & 0 \\
0 & 0 & 0 & 1
\end{array}\right)
$$

Let $\mathcal{D}$ be the global dephasing operation in the energy basis,

$$
\mathcal{D}(\cdot)=\sum_{E \in \operatorname{spec}\left(H_{C}+H_{H}\right)} \Pi_{E}(\cdot) \Pi_{E}
$$

where $\operatorname{spec}(X)$ is the spectrum of $X$ and $\Pi_{E}$ is the projector on the eigenspace of energy $E$. One can verify that, for all $U$ with $\left[U, H_{C}+H_{H}\right]=0$, neither $p^{\mathrm{TPM}}$ nor $p^{W}$ are affected by the application of $\mathcal{D}$ on the initial state $\rho_{C H}$. For the two-qubit case this implies that, without loss of generality, we can take the state with thermal marginals to have the form

$$
\rho_{C H}=\left(\begin{array}{cccc}
P_{00} & 0 & 0 & 0 \\
0 & \frac{1}{z_{C}}-P_{00} & \eta e^{i \xi} & 0 \\
0 & \eta e^{-i \xi} & \frac{1}{z_{H}}-P_{00} & 0 \\
0 & 0 & 0 & \frac{z_{C} z_{H}-z_{C}-z_{H}}{z_{C} z_{H}}+P_{00}
\end{array}\right),
$$

where $z_{C(H)}=1+e^{-\beta_{C(H)}}$. The two-qubit case must be resonant to have nontrivial dynamics, due to the fact that $U$ conserves energy (we discuss how to weaken this assumption later). We then set $H_{C}=H_{H}=|1\rangle\langle 1|$ by renormalizing the temperature. Since $\rho_{C H}$ has to be a valid density operator, $P_{00}$ must satisfy

$$
P_{00} \leq \frac{1}{z_{H}}
$$

$$
P_{00} \geq \frac{z_{C}+z_{H}-z_{C} z_{H}}{z_{C} z_{H}} .
$$

The MH and the TPM probabilities satisfy

$$
\begin{aligned}
& p^{W}{ }_{01,10}=p^{\mathrm{TPM}}{ }_{01,10}+\eta \cos \theta \sin \theta \cos \xi, \\
& p^{W}{ }_{10,01}=p^{\mathrm{TPM}}{ }_{10,01}-\eta \cos \theta \sin \theta \cos \xi,
\end{aligned}
$$




$$
\begin{aligned}
& p_{01,10}^{\mathrm{TPM}}=\left(\frac{1}{z_{C}}-P_{00}\right) \sin ^{2} \theta, \\
& p_{10,01}^{\mathrm{TPM}}=\left(\frac{1}{z_{H}}-P_{00}\right) \sin ^{2} \theta .
\end{aligned}
$$

The probabilities $p^{W}$ and $p^{\text {TPM }}$ are independent of $\kappa$; hence we set $\kappa=0$. Furthermore, $p^{\text {TPM }}$ are independent of $\lambda, \xi$, and $\phi$, while $p^{W}$ only depends upon them by a factor $\cos (\lambda+\xi+\phi)$. A simple reparametrization then allows one to set $\lambda=\phi=0$. One can also set $\xi=0$ by a redefinition of $\eta$. We can always take $\eta \geq 0$ by a reparametrization of $\theta$ (if $\eta \leq 0$ map $\theta \mapsto-\theta$ ). In the main text, we allow $\eta \in \mathbb{R}$ for an easier comparison to the experimental parameters of Ref. [51]. The final forms are

$$
U=\left(\begin{array}{cccc}
1 & 0 & 0 & 0 \\
0 & \cos (\theta) & -\sin (\theta) & 0 \\
0 & \sin (\theta) & \cos (\theta) & 0 \\
0 & 0 & 0 & 1
\end{array}\right),
$$

$$
\rho_{C H}=\left(\begin{array}{cccc}
P_{00} & 0 & 0 & 0 \\
0 & \frac{1}{z_{C}}-P_{00} & \eta & 0 \\
0 & \eta & \frac{1}{z_{H}}-P_{00} & 0 \\
0 & 0 & 0 & \frac{z_{C} z_{H}-z_{C}-z_{H}}{z_{C} z_{H}}+P_{00}
\end{array}\right),
$$

where $z_{C(H)}=1+e^{-\beta_{C(H)}}$ and $\eta \geq 0$.

A direct computation of the heat

$$
Q:=\operatorname{Tr}\left[\rho_{C H} H_{C}\right]-\operatorname{Tr}\left[U \rho_{C H} U^{\dagger} H_{C}\right]
$$

returns

$$
Q=-\eta \cos \xi \sin 2 \theta+\sin ^{2} \theta\left(\frac{1}{1+e^{\beta_{C}}}-\frac{1}{1+e^{\beta_{H}}}\right) .
$$

Note that $Q$ does not depend on $P_{00}$ and that $\rho_{C H} \geq 0$ implies $|\eta| \leq \sqrt{\left(1 / z_{C}-P_{00}\right)\left(1 / z_{H}-P_{00}\right)}$.

Concerning $Q^{\mathrm{TPM}}$,

$$
\left.Q^{\mathrm{TPM}}:=\operatorname{Tr}\left[\mathcal{D}\left(\rho_{C H}\right) H_{C}\right]\right)-\operatorname{Tr}\left[U \mathcal{D}\left(\rho_{C H}\right) U^{\dagger} H_{C}\right] .
$$

We have

$$
Q^{\mathrm{TPM}}=\sin ^{2} \theta\left(\frac{1}{1+e^{\beta_{C}}}-\frac{1}{1+e^{\beta_{H}}}\right) .
$$

Hence $Q^{\text {TPM }} \leq 0$, i.e., for the two-qubit case, no back flow exists in the TPM scheme. Note that, as is well known, $Q(\eta=0)=Q^{\mathrm{TPM}}$. That is, the average heat flows of the TPM scheme coincide with that of a state from which all initial coherence has been removed.

\section{b. Back flow and quantum correlations in two-qubit systems}

Next, we show that for a state that is diagonal in the energy basis (even if it includes classical correlations), no heat back flow can be observed. This can be checked by simply replacing $\eta=0$ in Eq. (B10), which gives

$$
Q(\eta=0)=Q^{\mathrm{TPM}} \leq 0 .
$$

Hence, $\eta \neq 0$ is necessary for back flow.

\section{Proof of Inequality 1}

Assume that we observe a direct flow, i.e., $Q<0$. From Eqs. (B6) and (B7), this that implies $\theta \neq 0$. Since we can then restrict to $\theta \in(0, \pi)$, Eq. (B12) and $\beta_{1} \neq \beta_{2}$ imply $Q^{\mathrm{TPM}}<0$. Hence, we can define $\alpha:=Q / Q^{\mathrm{TPM}}$. From Eq. (B6), we obtain

$$
2 p_{10,01}^{W}=(\alpha+1) p_{10,01}^{\mathrm{TPM}}-(\alpha-1) p_{01,10}^{\mathrm{TPM}} .
$$

We have $\alpha>0$ and, due to Eq. (B4), $p_{01,10}^{\mathrm{TPM}}>0$. Then $p_{10,01}^{W} \geq 0$ implies that

$$
\frac{p_{10,01}^{\mathrm{TPM}}}{p_{01,10}^{\mathrm{TPM}}} \geq \frac{\alpha-1}{\alpha+1}=\frac{Q-Q^{\mathrm{TPM}}}{Q+Q^{\mathrm{TPM}}} .
$$

We further note that, from Eq. (B7),

$$
\frac{p_{10,01}^{\mathrm{TPM}}}{p_{01,10}^{\mathrm{TPM}}}=\frac{z_{C}\left(1-z_{H} P_{00}\right)}{z_{H}\left(1-z_{C} P_{00}\right)} \leq \frac{1+e^{\beta_{H}}}{1+e^{\beta_{C}}},
$$

where equality is obtained for the minimal value of $P_{00}$ in Eq. (B5). From Eqs. (B14) and (B15), we have

$$
\frac{1+e^{\beta_{H}}}{1+e^{\beta_{C}}} \geq \frac{Q-Q^{\mathrm{TPM}}}{Q+Q^{\mathrm{TPM}}}
$$


which is the statement of Inequality 1 for $Q<0$.

In a similar manner, we can repeat the calculations for $Q>0$. In this case,

$$
2 p_{01,10}^{W}=(\alpha+1) p_{01,10}^{\mathrm{TPM}}-(\alpha-1) p_{10,01}^{\mathrm{TPM}} .
$$

Again, we have $Q^{\mathrm{TPM}}<0$, which implies $\alpha<0$, and $p_{10,01}^{\mathrm{TPM}}>0$. Hence $p_{01,10}^{W} \geq 0$ implies that

$$
\frac{p_{10,01}^{\mathrm{TPM}}}{p_{01,10}^{\mathrm{TPM}}} \geq \frac{\alpha+1}{\alpha-1}=\frac{Q+Q^{\mathrm{TPM}}}{Q-Q^{\mathrm{TPM}}}
$$

From Eqs. (B15) and (B18),

$$
\frac{1+e^{\beta_{H}}}{1+e^{\beta_{C}}} \leq \frac{Q+Q^{\mathrm{TPM}}}{Q-Q^{\mathrm{TPM}}}
$$

This is Inequality 1 in the case $Q>0$.

\section{Nonideal heat exchange: Inequality 2 \\ a. Assumptions and some consequences}

We find the tolerance of Inequality 1 to imperfections. The relevant assumptions are as follows:

A1 Rather than assuming that the dynamics $U$ on $\mathrm{CH}$ satisfy $\left[U, H_{C H}\right]=0$ (no work injected), we only assume that there exists some $\tilde{U}$ close to $U(\|U-\tilde{U}\| \leq \varepsilon)$ that satisfies $\left[\tilde{U}, H_{C H}\right]=0$ (small work injected).

A2 In the setting where no work is injected, the two local thermal states support heat exchanges only if they are resonant. We drop this assumption and define the local Hamiltonians $H_{C(H)}=E_{C(H)}|1\rangle\langle 1|$, where $E_{H}-E_{C}=\delta$ is how much they are off resonance. We assume that this gap is not too large: if the average energy is $\bar{E}=\frac{1}{2}\left(E_{H}+E_{C}\right)$,

$$
\Delta \equiv \frac{|\delta|}{2 \bar{E}} \leq \frac{e^{\beta_{C} E_{C}}-e^{\beta_{H} E_{H}}}{2+e^{\beta_{C} E_{C}}+e^{\beta_{H} E_{H}}} \equiv \frac{1-R}{1+R},
$$

where we define by $R$ the ratio $R=\left(1+e^{\beta_{H} E_{H}}\right) /(1+$ $\left.e^{\beta_{C} E_{C}}\right)$.

A3 Since some work is now injected, we will only consider energy flows that are far enough from zero that they cannot be simply due to work being injected directly into the local system by the driving. Our conclusions will hence be valid only for dynamics $U$ inducing energy flows

$$
|Q(U)| \geq 2 \bar{E} \varepsilon
$$

Above, $\|\cdot\|$ denotes the Shatten infinite norm

$$
\|A\|:=\max _{|v\rangle \text { s.t. }\|v\|=1} \| A|v\rangle \| \text {. }
$$

We will also use the Shatten 1-norm or trace norm

$$
\|A\|_{1}:=\operatorname{Tr}\left[\sqrt{A^{\dagger} A}\right] .
$$

Let us start with some inequalities that will be useful later and then deal with the two cases (direct flow and back flow) separately.

Using Hölder's inequality $\left|\operatorname{Tr}\left[B^{\dagger} A\right]\right| \leq\|B\|\|A\|_{1}$ and the submultiplicativity of the norm $\|A\|_{1}$,

$$
\begin{aligned}
& \left|p_{i_{C} i_{H} \rightarrow f_{C} f_{H}}^{W}(U)-p_{i_{C} i_{H} \rightarrow f_{C} f_{H}}^{W}(\tilde{U})\right| \\
& =\mid \operatorname{Re} \operatorname{Tr}\left[U^{\dagger}\left(\Pi^{f_{C} f_{H}}\right) U \Pi^{i_{C} i_{H}} \rho_{C H}\right] \\
& -\operatorname{Re} \operatorname{Tr}\left[\tilde{U}^{\dagger}\left(\Pi^{f_{C} f_{H}}\right) \tilde{U} \Pi^{i_{C} i_{H}} \rho_{C H}\right] \\
& \leq \frac{1}{2}\left\|U^{\dagger}-\tilde{U}^{\dagger}\right\|\left\|\Pi^{f_{C} f_{H}} U\left(\Pi^{i_{C} i_{H}} \rho_{C H}+\rho_{C H} \Pi^{i_{C} i_{H}}\right)\right\|_{1} \\
& +\frac{1}{2}\|U-\tilde{U}\|\left\|\Pi^{f_{C} f_{H}} \tilde{U}^{\dagger}\left(\Pi^{i_{C} i_{H}} \rho_{C H}+\rho_{C H} \Pi^{i_{C} i_{H}}\right)\right\|_{1} \\
& \leq 2\|U-\tilde{U}\|\left\|\Pi^{f_{C} f_{H}}\right\|_{1}\left\|\tilde{U}^{\dagger}\right\|_{1}\left\|\Pi^{i_{C} i_{H}}\right\|_{1}\left\|\rho_{C H}\right\|_{1} \\
& \leq 2\|U-\tilde{U}\| \leq 2 \varepsilon \text {. }
\end{aligned}
$$

In a similar manner, one can bound

$$
\left|p_{i_{C} i_{H} \rightarrow f_{C} f_{H}}^{\mathrm{TPM}}(U)-p_{i_{C} i_{H} \rightarrow f_{C} f_{H}}^{\mathrm{TPM}}(\tilde{U})\right| \leq 2 \varepsilon .
$$

Define $\tilde{p}^{W} \equiv p^{W}(\tilde{U})$ and $\tilde{p}^{\mathrm{TPM}} \equiv p^{\mathrm{TPM}}(\tilde{U})$. Using Eq. (B6), the heat under the energy-preserving unitary $\tilde{U}$ can be expressed as

$$
\begin{aligned}
Q(\tilde{U})= & \tilde{p}_{10,01}^{W} E_{H}-\tilde{p}_{01,10}^{W} E_{C} \\
= & \left(\tilde{p}_{10,01}^{W}-\tilde{p}_{01,10}^{W}\right) \bar{E}+\frac{1}{2}\left(\tilde{p}_{10,01}^{W}+\tilde{p}_{01,10}^{W}\right) \delta \\
= & \left(\tilde{p}_{10,01}^{W}-\tilde{p}_{01,10}^{W}\right) \bar{E} \\
& +\frac{1}{2}\left(\tilde{p}_{10,01}^{\mathrm{TPM}}+\tilde{p}_{01,10}^{\mathrm{TPM}}\right) \delta, \\
Q^{\mathrm{TPM}}(\tilde{U})= & \left(\tilde{p}_{10,01}^{\mathrm{TPM}}-\tilde{p}_{01,10}^{\mathrm{TPM}}\right) \bar{E} \\
& +\frac{1}{2}\left(\tilde{p}_{10,01}^{\mathrm{TPM}}+\tilde{p}_{01,10}^{\mathrm{TPM}}\right) \delta .
\end{aligned}
$$

From Eqs. (B24)-(B26),

$$
\begin{aligned}
&|Q(U)-Q(\tilde{U})| \leq 2 \bar{E} \varepsilon, \\
&\left|Q^{\mathrm{TPM}}(U)-Q^{\mathrm{TPM}}(\tilde{U})\right| \leq 2 \bar{E} \varepsilon .
\end{aligned}
$$

We will now study two cases separately. 


\section{b. Direct flow}

Following the same procedure as in Sec. 1, some algebra gives

$$
\begin{aligned}
2 \tilde{p}_{10,01}^{W}= & (\alpha+1) \tilde{p}_{10,01}^{\mathrm{TPM}}-(\alpha-1) \\
& \times\left(\tilde{p}_{01,10}^{\mathrm{TPM}}-\frac{\delta}{2 \bar{E}}\left(\tilde{p}_{10,01}^{\mathrm{TPM}}+\tilde{p}_{01,10}^{\mathrm{TPM}}\right)\right) .
\end{aligned}
$$

Using assumption (A3) and the fact that $Q(U) \leq 0$ (direct flow),

$$
Q(U)+2 \bar{E} \varepsilon \leq 0
$$

This and Eq. (B27) imply $Q(\tilde{U}) \leq 0$ and so $\alpha:=$ $Q(\tilde{U}) / Q^{\mathrm{TPM}}(\tilde{U}) \geq 0$. Assuming that $p^{W} \geq 0$, Eq. (B24) implies $2 \tilde{p}_{10,01}^{W}+4 \varepsilon \geq 0$. This allows us to derive from Eq. (B28) the inequality

$$
\begin{aligned}
& \frac{\tilde{p}_{10,01}^{\mathrm{TPM}}}{\tilde{p}_{01,10}^{\mathrm{TPM}}}-\frac{(\alpha-1)}{\alpha+1}+\frac{(\alpha-1)}{\alpha+1} \frac{\delta}{2 \bar{E}}\left(1+\frac{\tilde{p}_{10,01}^{\mathrm{TPM}}}{\tilde{p}_{01,10}^{\mathrm{TPM}}}\right) \\
& \quad+\frac{4 \varepsilon}{(\alpha+1) \tilde{p}_{01,10}^{\mathrm{TPM}}} \geq 0 .
\end{aligned}
$$

Recalling from Eq. (B15) that $\tilde{p}_{10,01}^{\mathrm{TPM}} / \tilde{p}_{01,10}^{\mathrm{TPM}} \leq\left(1+e^{\beta_{H} E_{H}}\right) /$ $\left(1+e^{\beta_{C} E_{C}}\right) \equiv R$,

$$
\begin{aligned}
Q(\tilde{U}) \geq & \frac{1+R-\Delta(1+R)}{1-R-\Delta(1+R)} Q^{\mathrm{TPM}}(\tilde{U}) \\
& +4 \varepsilon \frac{1-\Delta(1+R)}{1-R-\Delta(1+R)} \frac{Q^{\mathrm{TPM}}(\tilde{U})}{\tilde{p}_{01,10}^{\mathrm{TPM}}} \\
\geq & \frac{1+R-\Delta(1+R)}{1-R-\Delta(1+R)} Q^{\mathrm{TPM}}(\tilde{U}) \\
& -4 \bar{E} \varepsilon \frac{(1-\Delta(1+R))(1+\Delta)}{1-R-\Delta(1+R)} .
\end{aligned}
$$

In the first inequality, we use assumption (A2) and in the second we use the relation $Q^{\mathrm{TPM}}(\tilde{U}) / \tilde{p}_{01,10}^{\mathrm{TPM}} \geq-\bar{E}(1+$ $\Delta)$. Finally, expressing the results for the nonideal heat exchange, we apply Eq. (B27) to the last inequality and obtain for the direct flow

$$
\begin{aligned}
Q(U) \geq & \frac{1+R-\Delta(1+R)}{1-R-\Delta(1+R)} Q^{\mathrm{TPM}}(U) \\
& -4 \bar{E} \varepsilon \frac{(1-\Delta(1+R))(2-\Delta)}{1-R-\Delta(1+R)} .
\end{aligned}
$$

Note also that this weaker inequality holds:

$$
\begin{aligned}
Q(U) \geq & \frac{1+R-\Delta(1+R)}{1-R-\Delta(1+R)} Q^{\mathrm{TPM}}(U) \\
& -4 \bar{E} \varepsilon \frac{2+\Delta(1+R)}{1-R-\Delta(1+R)} .
\end{aligned}
$$

\section{c. Back flow}

Following a procedure as in Sec. 1, some algebra gives

$$
\begin{aligned}
2 \tilde{p}_{01,10}^{W}= & (\alpha+1) \tilde{p}_{01,10}^{\mathrm{TPM}}-(\alpha-1) \\
& \times\left(\tilde{p}_{10,01}^{\mathrm{TPM}}+\frac{\delta}{2 \bar{E}}\left(\tilde{p}_{01,10}^{\mathrm{TPM}}+\tilde{p}_{10,01}^{\mathrm{TPM}}\right)\right),
\end{aligned}
$$

Using assumption (A3) and the fact that $Q(U) \geq 0$ (back flow),

$$
Q(U)-2 \bar{E} \varepsilon \geq 0 .
$$

This and Eq. (B27) imply that $Q(\tilde{U}) \geq 0$ and $\alpha:=$ $Q(\tilde{U}) / Q^{\mathrm{TPM}}(\tilde{U}) \leq 0$. Assuming that $p^{W} \geq 0$, we have $2 \tilde{p}_{01,10}^{W}+4 \varepsilon \geq 0$. This relation gives

$$
\begin{aligned}
& \frac{\tilde{p}_{10,01}^{\mathrm{TPM}}}{\tilde{p}_{01,10}^{\mathrm{TPM}}}-\frac{\alpha+1}{\alpha-1}+\frac{\delta}{2 \bar{E}}\left(1+\frac{\tilde{p}_{10,01}^{\mathrm{TPM}}}{\tilde{p}_{01,10}^{\mathrm{TPM}}}\right) \\
& +\frac{4 \varepsilon}{(\alpha-1) \tilde{p}_{01,10}^{\mathrm{TPM}}} \geq 0 .
\end{aligned}
$$

As before, since $\tilde{p}_{10,01}^{\mathrm{TPM}} / \tilde{p}_{01,10}^{\mathrm{TPM}} \leq R$ and using assumption (A2), some algebra gives

$$
\begin{aligned}
Q(\tilde{U}) \leq & -\frac{1+R+\Delta(1+R)}{1-R-\Delta(1+R)} Q^{\mathrm{TPM}}(\tilde{U}) \\
& -4 \varepsilon \frac{1}{1-R-\Delta(1+R)} \frac{Q^{\mathrm{TPM}}(\tilde{U})}{\tilde{p}_{01,10}^{\mathrm{TPM}}} \\
\leq & \frac{1+R+\Delta(1+R)}{1-R-\Delta(1+R)} Q^{\mathrm{TPM}}(\tilde{U}) \\
& +4 \bar{E} \varepsilon \frac{1-R+\Delta(1+R)}{1-R-\Delta(1+R)},
\end{aligned}
$$

where we use the relation $-Q^{\mathrm{TPM}}(\tilde{U}) / \tilde{p}_{01,10}^{\mathrm{TPM}} \leq \bar{E}(1-R+$ $\Delta(1+R))$. Applying Eq. (B27),

$$
\begin{aligned}
Q(U) \leq & -\frac{1+R+\Delta(1+R)}{1-R-\Delta(1+R)} Q^{\mathrm{TPM}}(U) \\
& +4 \bar{E} \varepsilon \frac{(2-R+\Delta(1+R))}{1-R-\Delta(1+R)} .
\end{aligned}
$$

While the two separate inequalities are stronger, the directand back-flow inequalities for nonideal heat exchange in Eqs. (B33) and (B38) can also be cast into a compact, symmetric representation:

$$
\begin{aligned}
|Q| \leq & \frac{1+R+\Delta(1+R)}{1-R-\Delta(1+R)}\left|Q^{\mathrm{TPM}}\right| \\
& +4 \bar{E} \varepsilon \frac{2+\Delta(1+R)}{1-R-\Delta(1+R)} .
\end{aligned}
$$




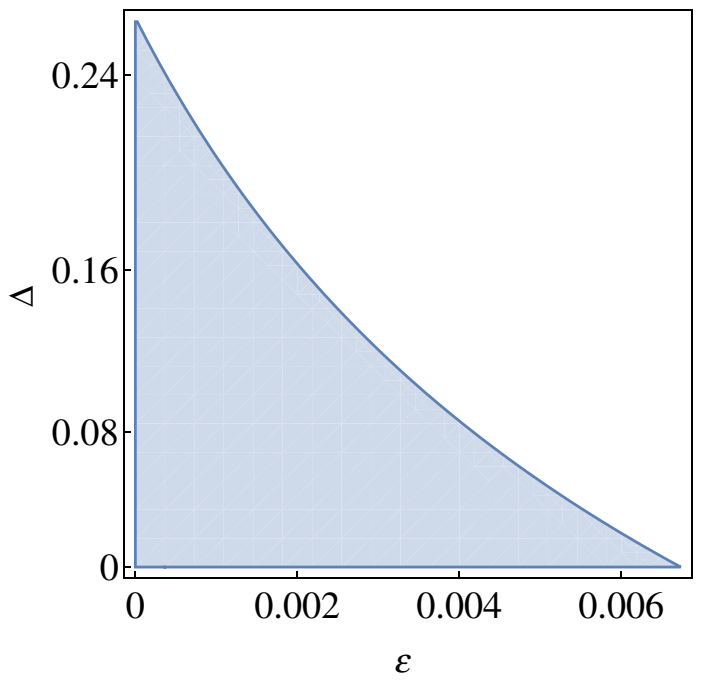

FIG. 6. The shaded area indicates the parameter regime in which Inequality 2 can be violated, i.e., negativity can be detected for nonideal heat-exchange processes. Here, $\omega_{C}=1.2$ $\mathrm{kHz}, J=220 \mathrm{~Hz}, T_{C}=3.48 \cdot 10^{-8} \mathrm{~K}, T_{H}=1.74 \cdot 10^{-7} \mathrm{~K}, \gamma=$ -0.19 , and $t=4 \mathrm{~ms}$.

\section{Nonideal heat exchange: example}

We study the violation of Inequality 2. Our starting point is the setup considered in the experiment in Ref. [51] with two modifications. First, we add a small perturbation, such that the unitary is no longer energy preserving. In particular, we add an interaction Hamiltonian of the form $J_{x} \sigma_{x}^{H} \sigma_{x}^{C}$ , where $J_{x}$ determines the distance between the unitaries $\varepsilon$. Second, the two qubits have different Hamiltonians that are characterized by $\Delta=\left(\omega_{H}-\omega_{C}\right) /\left(\omega_{H}+\omega_{C}\right)$. In Fig. 6, we present the tolerance of our inequality to nonideal heat exchange. The shaded area indicates the violation of Inequality 2 as a function of $\varepsilon$ and $\Delta$.

\section{APPENDIX C: EXTENSIONS TO ARBITRARY FINITE-DIMENSIONAL SYSTEMS}

\section{Proof of the Quantum XFT}

Here, we provide a proof of the quantum XFT in the main text. Traditionally, fluctuation theorems are derived by considering the ratio of the forward- and backwardprocess probabilities $[1,4]$. Hence, we consider

$$
\frac{p_{i_{C} i_{H} \rightarrow f_{C} f_{H}}^{W}}{\tilde{p}_{f_{C} f_{H} \rightarrow i_{C} i_{H}}^{W}}=\frac{\operatorname{Re} \operatorname{Tr}\left[\mathcal{U}^{\dagger}\left(\Pi^{f_{C} f_{H}}\right) \rho^{i_{C} i_{H}}\right]}{\operatorname{Re} \operatorname{Tr}\left[\mathcal{U}\left(\Pi^{i_{C} i_{H}}\right) \rho_{C} f_{H}\right]} e^{-\Delta I-\Delta \beta \Delta E_{i} f_{C}},
$$

where $\tilde{p}_{f_{C H} \rightarrow i_{C} i_{H}}^{W}:=\operatorname{Re} \operatorname{Tr}\left[\mathcal{U}\left(\Pi^{i_{C} i_{H}}\right) \Pi^{f_{C} f_{H}} \rho_{C H}\right], \rho^{i_{C} i_{H}}=$ $\Pi^{i_{C} i_{H}} \rho_{C H} / \operatorname{Tr}\left[\Pi^{i{ }^{i} i_{H}} \rho_{C H}\right]$, and $\Delta I:=I_{f_{C} f_{H}}-I_{i_{C} i_{H}}$, with

$$
I_{i_{C} i_{H}}=\log \operatorname{Tr}\left[\Pi^{i_{C} i_{H}} \rho_{C H}\right] / \operatorname{Tr}\left[\Pi^{i_{C}} \rho_{C}\right] \operatorname{Tr}\left[\Pi^{i_{H}} \rho_{H}\right],
$$

the elements of the classical mutual information. Note that we assume that $E_{i_{C}}-E_{f_{C}} \approx E_{f_{H}}-E_{i_{H}}$ for all nonzero $p^{W}{ }_{i_{C} i_{H} \rightarrow f_{C} f_{H}}$. One can then obtain the quantum XFT from Eq. (C1) by multiplying both sides by $\tilde{p}^{W} f_{C f_{H} \rightarrow i_{C} i_{H}} e^{\Delta I+\Delta \beta \Delta E_{i} f_{C}}$ and summing over all indexes.

\section{Alternative bound to Inequality 3}

Here, we derive an alternative bound (Inequality 4) for the detection of negativity. However, we note that, for all the case studies considered, we find Inequality 3 to be superior to Inequality 4.

A direct calculation of $\sum p_{i_{C} i_{H} \rightarrow f_{C} f_{H}} e^{\Delta \beta \Delta E i_{C} f_{C}}$ leads to the following:

$$
\begin{aligned}
\left\langle e^{\Delta \beta \Delta E i_{C} f_{C}}\right\rangle & =1+J \\
& =1+\operatorname{Re} \operatorname{Tr}\left[\mathcal{U}^{\dagger}\left(\rho_{C} \otimes \rho_{H}\right)(c(\rho)+q(\rho))\right],
\end{aligned}
$$

where

$$
c(\rho)=\sum_{i_{C} i_{H}} \frac{\Pi^{i_{C} i_{H}}\left(\rho_{C H}-\rho_{C} \otimes \rho_{H}\right) \Pi^{i_{C} i_{H}}}{\operatorname{Tr}\left[\Pi^{i} \rho_{C}\right] \operatorname{Tr}\left[\Pi^{i_{H}} \rho_{H}\right]},
$$

$$
q(\rho)=\sum_{i_{C} i_{H}} \frac{\Pi^{i_{C} i_{H}} \rho_{C H} \Pi^{i_{C} i_{H} \perp}}{\operatorname{Tr}\left[\Pi^{i_{C}} \rho_{C}\right] \operatorname{Tr}\left[\Pi^{i_{H}} \rho_{H}\right]} .
$$

Note that $c\left(\rho_{C H}\right)=0$ if the populations of $\rho_{C H}$ coincide with those of $\rho_{C} \otimes \rho_{H}$, even if $\rho_{C H}$ has coherence. This is why we use the notation " $c$ " to indicate "classical correlations." On the other hand, $q\left(\rho_{C H}\right)=0$ whenever there is no coherence, even in the presence of classical correlations; hence the notation " $q$." The term 1 in Eq. (C3) is obtained noting that

$$
\begin{gathered}
\operatorname{Re} \operatorname{Tr}\left[\mathcal{U}^{\dagger}\left(\rho_{C} \otimes \rho_{H}\right)\left(\sum_{i_{C} i_{H}} \frac{\Pi^{i_{C} i_{H}}\left(\rho_{C} \otimes \rho_{H}\right) \Pi^{i_{C} i_{H}}}{\operatorname{Tr}\left[\Pi^{i_{C}} \rho_{C}\right] \operatorname{Tr}\left[\Pi^{i_{H}} \rho_{H}\right]}\right)\right] \\
=\operatorname{Re} \operatorname{Tr}\left[\mathcal{U}^{\dagger}\left(\rho_{C} \otimes \rho_{H}\right)\left(\sum_{i_{C} i_{H}} \Pi^{i_{C} i_{H}}\right)\right]=1 .
\end{gathered}
$$

Inequality 5. Let $\rho_{C H}$ be an arbitrary finite-dimensional system with thermal marginals $\left(\beta_{C} \neq \beta_{H}\right)$ and let $U$ be an energy-preserving unitary, $\left[U, H_{C}+H_{H}\right]=0$. If $p^{W}$ is non-negative,

$$
Q \leq(\Delta \beta)^{-1} \log (1+J) .
$$

The result is an application of Jensen's inequality to Eq. (C3). Using norm inequalities, it can be shown that

$$
J \leq\left\|c\left(\rho_{C H}\right)\right\|+\left\|q\left(\rho_{C H}\right)\right\|,
$$

so the right-hand side is finite. 


\section{Heat flow between two qudits}

To study the heat flow between two qudits, we first consider the case of two qutrits, which can then be generalized easily to $d$-dimensional systems. Setting $E_{0}=$ 0 , we take $H_{C}=H_{H}=\sum_{n=1}^{2} E_{n}|n\rangle\langle n|$ and assume no degeneracy of the energy gaps (the "Bohr spectrum") throughout this section (see Fig. 7). By the same reasoning as in the two-qubit scenario, for the purpose of studying heat flows we can reduce a general two-qutrit state to the form

$$
\rho_{C H}=\left(\begin{array}{ccccccccc}
\rho_{0} & 0 & 0 & 0 & 0 & 0 & 0 & 0 & 0 \\
0 & \rho_{1} & 0 & \rho_{13} & 0 & 0 & 0 & 0 & 0 \\
0 & 0 & \rho_{2} & 0 & 0 & 0 & \rho_{26} & 0 & 0 \\
0 & \rho_{31} & 0 & \rho_{3} & 0 & 0 & 0 & 0 & 0 \\
0 & 0 & 0 & 0 & \rho_{4} & 0 & 0 & 0 & 0 \\
0 & 0 & 0 & 0 & 0 & \rho_{5} & 0 & \rho_{57} & 0 \\
0 & 0 & \rho_{62} & 0 & 0 & 0 & \rho_{6} & 0 & 0 \\
0 & 0 & 0 & 0 & 0 & \rho_{75} & 0 & \rho_{7} & 0 \\
0 & 0 & 0 & 0 & 0 & 0 & 0 & 0 & \rho_{8}
\end{array}\right),
$$

where $\rho_{i j}=\rho_{j i}^{*}=\eta_{i j} e^{-i \xi_{i j}} \sqrt{\rho_{i} \rho_{j}}$, with $\eta_{\mathrm{ij}} \in[0,1], \xi_{i j} \in \mathbb{R}$, and we use the natural labeling $(00,01,02,10,11,12, \ldots)$ $\equiv(0,1,2,3,4,5, \ldots)$. If we impose the constraints $\operatorname{Tr}\left[\rho_{C H}\right]=1$ and $\operatorname{Tr}_{C(H)}\left[\rho_{C H}\right]=\rho_{H(C)}$, with

$$
\rho_{X}=\left(\begin{array}{ccc}
1 & 0 & 0 \\
0 & e^{-\beta_{X} E_{1}} & 0 \\
0 & 0 & e^{-\beta_{X} E_{2}}
\end{array}\right) \operatorname{Tr}\left[\rho_{X}\right]^{-1},
$$

we are left with the free parameters $\eta_{i j}$, $\xi_{i j}$ and four undetermined populations $\rho_{i}$ that must comply with the non-negativity of $\rho_{C H}$.

The general energy-preserving unitary takes the form

$$
U=\left(\begin{array}{ccccccccc}
1 & 0 & 0 & 0 & 0 & 0 & 0 & 0 & 0 \\
0 & e^{i\left(\kappa_{1}+\lambda_{01}\right)} c_{01} & 0 & -e^{i\left(\kappa_{1}-\phi_{01}\right)} s_{01} & 0 & 0 & 0 & 0 & 0 \\
0 & 0 & e^{i\left(\kappa_{2}+\lambda_{02}\right)} c_{02} & 0 & 0 & 0 & -e^{i\left(\kappa_{2}-\phi_{02}\right)} s_{02} & 0 & 0 \\
0 & e^{i\left(\kappa_{1}+\phi_{01}\right)} s_{01} & 0 & e^{i\left(\kappa_{1}-\lambda_{01}\right)} c_{01} & 0 & 0 & 0 & 0 & 0 \\
0 & 0 & 0 & 0 & e^{i \kappa_{3}} & 0 & 0 & 0 & 0 \\
0 & 0 & 0 & 0 & 0 & e^{i\left(\kappa_{4}+\lambda_{12}\right)} c_{12} & 0 & -e^{i\left(\kappa_{4}-\phi_{12}\right)} s_{12} & 0 \\
0 & 0 & e^{i\left(\kappa_{2}+\phi_{02}\right)} s_{02} & 0 & 0 & 0 & e^{i\left(\kappa_{2}-\lambda_{02}\right)} c_{02} & 0 & 0 \\
0 & 0 & 0 & 0 & 0 & e^{i\left(\kappa_{4}-\phi_{12}\right)} s_{12} & 0 & e^{i\left(\kappa_{4}-\lambda_{12}\right)} c_{12} & 0 \\
0 & 0 & 0 & 0 & 0 & 0 & 0 & 0 & e^{i \kappa 5}
\end{array}\right) .
$$

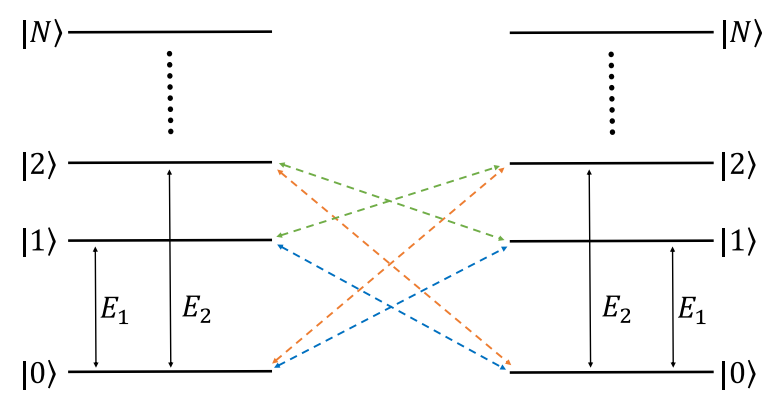

FIG. 7. A schematic of the energy levels of the two qudits and the couplings between the two.
Here, we use the short-cut notation $c_{n m}:=\cos \left(\theta_{n m}\right)$ and $s_{n m}:=\sin \left(\theta_{n m} t\right)$, where $\theta_{n m}=\theta_{m n}, \phi_{n m}=\phi_{m n}$ and $\lambda_{n m}=$ $\lambda_{m n}$ are parameters characterizing the coupling between the $n$th level of the first qutrit and the $m$ th level of the second qutrit. Within the manifolds $(01,02,12)$, $\kappa$ are global phases that do not change the energytransfer transition probabilities. One can note the similarity between the $U$ for the qutrits with that of the qubits. The heat-flow probabilities can be split into contributions from independent manifolds $(01,02,12)$ that possess the same structure as seen in Eq. (B1). The generalization to higher dimensions is now straightforward, as 
the structure of $\rho_{C H}$ and $U$ in Eqs. (C8) and (C10) is preserved.

We can express the transition probability that is related to heat transfer for arbitrary finite dimensions with nondegenerate gaps as

$$
\begin{aligned}
\forall E_{n}> & E_{m}, \quad p_{n m \rightarrow m n}^{W} \\
= & \rho_{n d+m} \sin ^{2}\left(\theta_{n m}\right)-\frac{1}{2} \eta_{n m} \sqrt{\rho_{n d+m} \rho_{m d+n}} \sin \left(2 \theta_{n m}\right) \\
& \times \cos \left(\xi_{n m}+\phi_{n m}+\lambda_{n m}\right) \\
\forall E_{n}< & E_{m}, \quad p_{n m \rightarrow m n}^{W} \\
= & \rho_{m d+n} \sin ^{2}\left(\theta_{n m}\right)+\frac{1}{2} \eta_{n m} \sqrt{\rho_{n d+m} \rho_{m d+n}} \sin \left(2 \theta_{n m}\right) \\
& \times \cos \left(\xi_{n m}+\phi_{n m}+\lambda_{n m}\right) .
\end{aligned}
$$

In a similar manner, we can calculate the transition probabilities obtained from the TPM scheme:

$$
\begin{array}{ll}
\forall E_{n}>E_{m}, & p_{n m \rightarrow m n}^{\mathrm{TPM}}=\rho_{n d+m} \sin ^{2}\left(\theta_{n m}\right) \\
\forall E_{n}<E_{m}, & p_{n m \rightarrow m n}^{\mathrm{TPM}}=\rho_{m d+n} \sin ^{2}\left(\theta_{n m}\right) .
\end{array}
$$

Here, we set $E_{0}=0$ and order the levels such that $E_{0} \leq$ $E_{1} \leq E_{2} \leq \cdots$ and $d$ is the dimension of the qudit. Next, we show that if all quasiprobabilities that contribute to direct or back flow are negative, then necessarily $|Q|>$ $\left|Q^{\mathrm{TPM}}\right|$. The difference $\Delta Q=Q-Q^{\mathrm{TPM}}$ reads

$$
\begin{aligned}
\Delta Q= & -\sum_{E_{n}>E_{m}} \eta_{n m} \sqrt{\rho_{n d+m} \rho_{m d+n}} \sin \left(2 \theta_{n m}\right) \\
& \times \cos \left(\xi_{n m}+\phi_{n m}+\lambda_{n m}\right)\left(E_{n}-E_{m}\right) .
\end{aligned}
$$

For the direct flow $(Q<0)$, if $\forall E_{n}>E_{m}$ we have $p_{n m \rightarrow m n}^{W}<0$, then from Eq. (C11) we immediately conclude that $\Delta Q<0$. For the back flow $(Q>0)$, if $\forall E_{n}<E_{m}$ we have $p_{n m \rightarrow m n}^{W}<0$, then from Eq. (C11) we now have that $\Delta Q>0$, which is exactly what we wanted to prove. Note that Eq. (C13) also suggests a way of maximizing the difference $\Delta Q$ for a given initial state. Choosing a protocol such that $\xi_{n m}+\lambda_{n m}=-\phi_{n m}$ and $\theta_{n m}= \pm \pi / 4$ for all $n, m$, we obtain

$$
|\Delta Q|_{\max }=\sum_{E_{n}>E_{m}} \eta_{n m} \sqrt{\rho_{n d+m} \rho_{m d+n}}\left(E_{n}-E_{m}\right) .
$$

In Fig. 8, we consider the two-qutrit setup of Eq. (C8). We plot the negativity in the direct flow and compare it to the violations of Inequalities 3 and 5 for different protocols. The protocols are determined by varying $\theta_{01}$ and $\theta_{02}$.

\section{Proof of inequality 4}

We define $\psi_{n m}=\xi_{n m}+\phi_{n m}+\lambda_{n m}$ and $\Delta E_{n m}=E_{n}-$ $E_{m}$. Using Eqs. (C11) and (C12), the symmetry $\theta_{n m}=\theta_{m n}$,

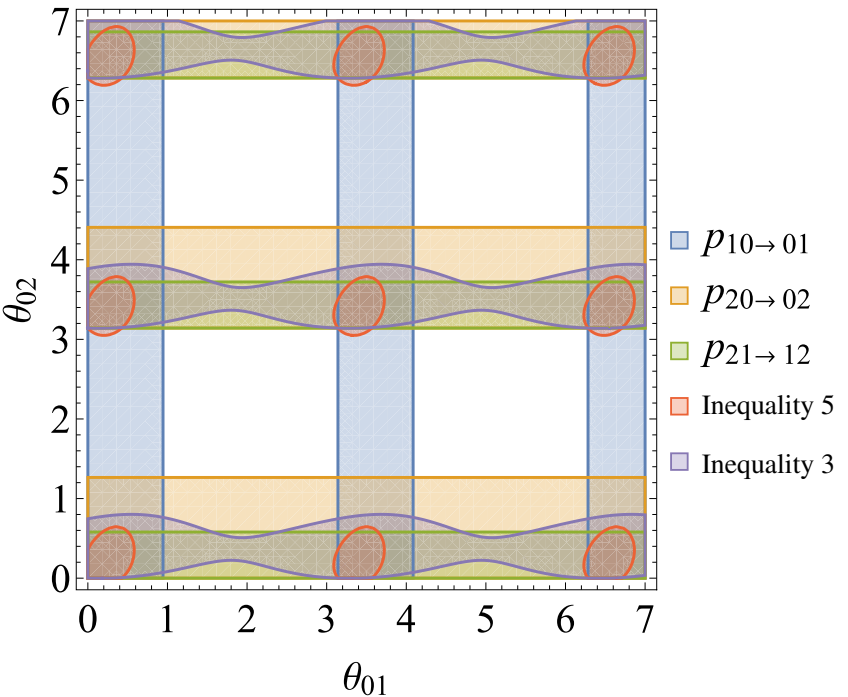

FIG. 8. The direct-flow negativity of the probabilities specified in the figure and the violation of Inequalities 3 and 5 for different interaction protocols determined by $\theta_{01}$ and $\theta_{02}$. Parameters: $\beta_{H}=0.3, \beta_{C}=1.3, \theta_{12}=\theta_{02}, E_{1}=1, E_{2}=1.15, \xi=\phi=\lambda=$ $0, \eta_{i j}=1$ for $(i, j)=\{(1,3),(2,6),(5,7)\}, \rho_{0}=0.3, \rho_{5}=0.03$, $\rho_{7}=0.07$, and $\rho_{8}=0.06$.

$\psi_{n m}=\psi_{m n}$, and noting that

$$
\begin{aligned}
Q & =\sum_{E_{n}>E_{m}} p_{n m \rightarrow m n}^{W} \Delta E_{n m}+\sum_{E_{n}<E_{m}} p_{n m \rightarrow m n}^{W} \Delta E_{n m} \\
& =\sum_{E_{n}>E_{m}} p_{n m \rightarrow m n}^{W} \Delta E_{n m}-\sum_{E_{n}>E_{m}} p_{m n \rightarrow n m}^{W} \Delta E_{n m},
\end{aligned}
$$

using $Q^{\mathrm{TPM}}=Q(\eta=0)$ the heat exchanged can be expressed as

$$
\begin{aligned}
Q= & Q^{\mathrm{TPM}} \\
& -\sum_{E_{n}>E_{m}} \eta_{n m} \sqrt{\rho_{n d+m} \rho_{m d+n}} \sin \left(2 \theta_{n m}\right) \cos \left(\psi_{n m}\right) \Delta E_{n m} .
\end{aligned}
$$

Using Eqs. (C11) and (C12) for $E_{n}>E_{m}$, non-negativity implies that

$$
2 p_{n m \rightarrow m n}^{\mathrm{TPM}}>\eta_{n m} \sqrt{\rho_{n d+m} \rho_{m d+n}} \sin \left(2 \theta_{n m}\right) \cos \left(\psi_{n m}\right) .
$$

We conclude that

$$
Q>Q^{\mathrm{TPM}}-2 \sum_{E_{n}>E_{m}} p_{n m \rightarrow m n}^{\mathrm{TPM}} \Delta E_{n m} .
$$

Violation of this inequality witnesses negativity in the direct flow, as illustrated by the upper-right process of Fig. 1 and proves the lower bound of inequality 4 . The 
upper bound on the heat can be obtained in a similar manner. To see this, note that the heat can be written as

$$
\begin{aligned}
Q= & Q^{\mathrm{TPM}}+\sum_{E_{n}<E_{m}} \eta_{n m} \sqrt{\rho_{n d+m} \rho_{m d+n}} \sin \left(2 \theta_{n m}\right) \\
& \times \cos \left(\psi_{n m}\right) \Delta E_{m n} .
\end{aligned}
$$

Using the non-negativity of Eq. (C11) for $E_{n}<E_{m}$, we have

$$
Q<Q^{\mathrm{TPM}}+2 \sum_{E_{n}<E_{m}} p_{n m \rightarrow m n}^{\mathrm{TPM}} \Delta E_{m n} .
$$

Violation of this inequality witnesses negativity in the back flow as illustrated by the lower-right process of Fig. 1 and proves the upper bound of Inequality 4.

[1] C. Jarzynski and D. K. Wójcik, Classical and Quantum Fluctuation Theorems for Heat Exchange, Phys. Rev. Lett. 92, 230602 (2004).

[2] S. Lloyd, Use of Mutual Information to Decrease Entropy: Implications for the Second Law of Thermodynamics, Phys. Rev. A 39, 5378 (1989).

[3] D. Jennings and T. Rudolph, Entanglement and the Thermodynamic Arrow of Time, Phys. Rev. E 81, 061130 (2010).

[4] S. Jevtic, T. Rudolph, D. Jennings, Y. Hirono, S. Nakayama, and M. Murao, Exchange fluctuation theorem for correlated quantum systems, Phys. Rev. E 92, 042113 (2015).

[5] M. Perarnau-Llobet, E. Bäumer, K. V. Hovhannisyan, M. Huber, and A. Acin, No-Go Theorem for the Characterization of Work Fluctuations in Coherent Quantum Systems, Phys. Rev. Lett. 118, 070601 (2017).

[6] M. Lostaglio, Quantum Fluctuation Theorems, Contextuality, and Work Quasiprobabilities, Phys. Rev. Lett. 120, 040602 (2018).

[7] S. Deffner, J. P. Paz, and W. H. Zurek, Quantum work and the thermodynamic cost of quantum measurements, Phys. Rev. E 94, 010103 (2016).

[8] K. Beyer, K. Luoma, and W. T. Strunz, Work as an external quantum observable and an operational quantum jarzynski equality, arXiv:2003.06437 (2020).

[9] A. Sone, Y.-X. Liu, and P. Cappellaro, Quantum jarzynski equality of open quantum systems in one-time measurement scheme, arXiv:2002.06332 (2020).

[10] E. Bäumer, M. Lostaglio, M. Perarnau-Llobet, and R. Sampaio, in Thermodynamics in the Quantum Regime: Fundamental Aspects and New directions, edited by F. Binder, L. A. Correa, C. Gogolin, J. Anders, and G. Adesso (Springer International Publishing, Cham, 2018), p. 275.

[11] A. E. Allahverdyan, Nonequilibrium quantum fluctuations of work, Phys. Rev. E 90, 032137 (2014).

[12] P. Solinas and S. Gasparinetti, Full distribution of work done on a quantum system for arbitrary initial states, Phys. Rev. E 92, 042150 (2015).

[13] P. Solinas and S. Gasparinetti, Probing Quantum Interference Effects in the Work Distribution, Phys. Rev. A 94, 052103 (2016).
[14] B.-M. Xu, J. Zou, L.-S. Guo, and X.-M. Kong, Effects of Quantum Coherence on Work Statistics, Phys. Rev. A 97, 052122 (2018).

[15] H. J. Miller and J. Anders, Time-reversal symmetric work distributions for closed quantum dynamics in the histories framework, New J. Phys. 19, 062001 (2017).

[16] S. Goldstein and D. N. Page, Linearly Positive Histories: Probabilities for a Robust Family of Sequences of Quantum Events, Phys. Rev. Lett. 74, 3715 (1995).

[17] R. Sampaio, S. Suomela, T. Ala-Nissila, J. Anders, and T. Philbin, The impossible quantum work distribution, arXiv:1707.06159 (2017).

[18] K. Micadei, G. T. Landi, and E. Lutz, Quantum Fluctuation Theorems Beyond Two-Point Measurements, Phys. Rev. Lett. 124, 090602 (2020).

[19] S. Gherardini, A. Belenchia, M. Paternostro, and A. Trombettoni, The role of quantum coherence in energy fluctuations, arXiv:2006.06208 [quant-ph] (2020).

[20] M. Campisi, P. Hänggi, and P. Talkner, Colloquium: Quantum fluctuation relations: Foundations and applications, Rev. Mod. Phys. 83, 771 (2011).

[21] M. Esposito, U. Harbola, and S. Mukamel, Nonequilibrium fluctuations, fluctuation theorems, and counting statistics in quantum systems, Rev. Mod. Phys. 81, 1665 (2009).

[22] P. P. Hofer, Quasi-probability distributions for observables in dynamic systems, Quantum 1, 32 (2017).

[23] P. Solinas and S. Gasparinetti, Probing Quantum Interference Effects in the Work Distribution, Phys. Rev. A 94, 052103 (2016).

[24] W. Belzig and Y. V. Nazarov, Full Counting Statistics of Electron Transfer Between Superconductors, Phys. Rev. Lett. 87, 197006 (2001).

[25] A. Bednorz and W. Belzig, Quasiprobabilistic Interpretation of Weak Measurements in Mesoscopic Junctions, Phys. Rev. Lett. 105, 106803 (2010).

[26] K. V. Hovhannisyan and A. Imparato, Quantum current in dissipative systems, New J. Phys. 21, 052001 (2019).

[27] A. Serafini, Quantum Continuous Variables: A Primer of Theoretical Methods (CRC Press, Boca Raton, FL, 2017).

[28] E. Wigner, On the quantum correction for thermodynamic equilibrium, Phys. Rev. 40, 749 (1932).

[29] H. Pashayan, J. J. Wallman, and S. D. Bartlett, Estimating Outcome Probabilities of Qquantum Circuits Using Quasiprobabilities, Phys. Rev. Lett. 115, 070501 (2015).

[30] J. R. Seddon, B. Regula, H. Pashayan, Y. Ouyang, and E. T. Campbell, Quantifying quantum speedups: improved classical simulation from tighter magic monotones, arXiv:2002.06181 (2020).

[31] N. Yunger Halpern, Jarzynski-Like Qquality for the Out-ofTime-Ordered Correlator, Phys. Rev. A 95, 012120 (2017).

[32] J. R. González Alonso, N. Yunger Halpern, and J. Dressel, Out-of-Time-Ordered-Correlator Quasiprobabilities Robustly Witness Scrambling, Phys. Rev. Lett. 122, 040404 (2019).

[33] Interestingly, Wigner originally introduced his function to compute quantum corrections to thermodynamic quantities.

[34] R. Kunjwal, M. Lostaglio, and M. F. Pusey, Anomalous Weak Values and Contextuality: Robustness, Tightness, and Imaginary Parts, Phys. Rev. A 100, 042116 (2019).

[35] J. Aberg, Quantifying Superposition, arXiv:0612146 (2006). 
[36] I. Marvian and R. W. Spekkens, How to Quantify Coherence: Distinguishing Speakable and Unspeakable Notions, Phys. Rev. A 94, 052324 (2016).

[37] Ref. [11] [Eq. (41)] or [55] (Theor. 7.5).

[38] H. Margenau and R. N. Hill, Correlation between measurements in quantum theory, progress of theoretical physics, Prog. Theor. Phys. 26, 722 (1961).

[39] Y. P. Terletsky, The limiting transition from quantum to classical mechanics, J. Exper. Theor. Phys. 7, 1290 (1937).

[40] J. Dressel, Weak Values as Interference Phenomena, Phys. Rev. A 91, 032116 (2015).

[41] Y. Aharonov, D. Z. Albert, and L. Vaidman, How the Result of a Measurement of a Component of the Spin of a Spin-1/2 Particle can Turn Out to be 100, Phys. Rev. Lett. 60, 1351 (1988).

[42] J. Dressel, M. Malik, F. M. Miatto, A. N. Jordan, and R. W. Boyd, Colloquium, Rev. Mod. Phys. 86, 307 (2014).

[43] H. M. Wiseman, Weak Values, Quantum Trajectories, and the Cavity-Qed Experiment on Wave-Particle Correlation, Phys. Rev. A 65, 032111 (2002).

[44] J. Dressel, S. Agarwal, and A. N. Jordan, Contextual Values of Observables in Quantum Measurements, Phys. Rev. Lett. 104, 240401 (2010).

[45] M. F. Pusey, Anomalous Weak Values are Proofs of Contextuality, Phys. Rev. Lett. 113, 200401 (2014).

[46] R. W. Spekkens, Contextuality for Preparations, Transformations, and Unsharp Measurements, Phys. Rev. A 71, 052108 (2005).
[47] R. W. Spekkens, Negativity and Contextuality are Equivalent Notions of Nonclassicality, Phys. Rev. Lett. 101, 020401 (2008).

[48] This follows from the fact that $\left[U^{\dagger} H_{C H} U, H_{C H}\right] \propto 1 / N$.

[49] S. Lloyd, Z.-W. Liu, S. Pirandola, V. Chiloyan, Y. Hu, S. Huberman, and G. Chen, No energy transport without discord, arXiv:1510.05035 (2015).

[50] C. Jarzynski, H. T. Quan, and S. Rahav, Quantum-Classical Correspondence Principle for Work Distributions, Phys. Rev. X 5, 031038 (2015).

[51] K. Micadei, J. P. Peterson, A. M. Souza, R. S. Sarthour, I. S. Oliveira, G. T. Landi, T. B. Batalhão, R. M. Serra, and E. Lutz, Reversing the direction of heat flow using quantum correlations, Nat. Commun. 10, 2456 (2019).

[52] S. Pal, T. S. Mahesh, and B. K. Agarwalla, Experimental Demonstration of the Validity of the Quantum HeatExchange Fluctuation Relation in an nmr Setup, Phys. Rev. A 100, 042119 (2019).

[53] H. Kwon and M. S. Kim, Fluctuation Theorems for a Quantum Channel, Phys. Rev. X 9, 031029 (2019).

[54] Note that the term in Eq. (16) may diverge as $\rho_{k k} \rightarrow 0$. In Appendix $\mathrm{C}$, we derive a different bound for the heat in Eq. (17) that bypasses this problem. Also note that neither $\langle\Delta I\rangle_{\mathrm{W}}$ nor $\langle\Delta I\rangle_{T} \mathrm{PM}$ admit a simple interpretation as the change in classical mutual information in the energetic degrees of freedom.

[55] F. Zhang, , Matrix Theory: Basic Results and Techniques (Springer Science \& Business Media, New York, 2011).

[56] S. Wu and K. Mølmer, Weak Measurements With a Qubit Meter, Phys. Lett. A 374, 34 (2009). 